\title{
Klimaanpassung in der Bauleitplanung. Zum Integrationsstand klimaanpassungsrelevanter Maßnahmen in Flächennutzungs- und Bebauungsplänen mittelgroßer Städte Deutschlands
}

\author{
Bettina Huber (1), Lea Dunst (1) \\ Eingegangen: 22. Oktober 2020 - Angenommen: 31. Mai 2021 - Online veröffentlicht: 14. Juni 2021
}

\begin{abstract}
Zusammenfassung
Städte und Gemeinden haben eine zentrale Rolle bei der erfolgreichen Umsetzung von Klimaanpassungsbestrebungen, jedoch ist unklar, in welchem Ausmaß die kommunalen Planungsinstrumente die derzeit bestehenden Handlungsmöglichkeiten ausschöpfen. Forschungsgegenstand des vorliegenden Beitrags ist der aktuelle Stand der Integration von Klimaanpassungsmaßnahmen in die kommunale Bauleitplanung. Flächennutzungspläne und Bebauungspläne von zehn mittelgroßen deutschen Städte werden auf 24 verschiedene Anpassungsmaßnahmen aus sechs verschiedenen Handlungsfeldern hin analysiert. Außerdem werden beide Pläne auf inhaltliche Kohärenz untersucht. Die Untersuchung basiert auf einer qualitativen strukturierenden Inhaltsanalyse von insgesamt 81 Planungsdokumenten, deren Ergebnisse im Nachgang quantitativ ausgewertet wurden. Die Ergebnisse zeigen ein unausgeschöpftes Potenzial auf beiden Planungsebenen. Die größten Erfolge zeigen verschiedene Low-regret-Maßnahmen mit Bezug zu Stadtgrün.
\end{abstract}

Bettina Huber, adelphi, Alt-Moabit 91, 10559 Berlin, Deutschland b.huber@adelphi.de

Lea Dunst, adelphi, Alt-Moabit 91, 10559 Berlin, Deutschland lea-dunst@hotmail.de

(c) (1) $(92021$ Huber; licensee oekom verlag. This Open Access article is published under a Creative Commons Attribution 4.0 International License.
Schlüsselwörter: Bauleitplanung - Flächennutzungsplan • Bebauungsplan - Klimawandel - Klimaanpassung Anpassungskapazität

Climate Change Adaptation in Urban Land Use Planning. On the integration status of climate adaptation-relevant measures in land-use plans and local development plans of mediumsized cities in Germany

\begin{abstract}
Cities and municipalities play a key role in the successful implementation of climate adaptation efforts, but it is unclear to what extent municipal planning instruments exploit their existing potentials. The research subject of the present article is therefore the current status of the integration of climate adaptation measures into urban land-use planning. Land-use plans and local development plans of ten medium-sized German cities are analysed for 24 different adaptation measures from six different fields of action. In addition, both levels of urban land-use planning were analysed for coherence in terms of content. The study is based on a qualitative structuring content analysis of in total 81 planning documents, using a category-based coding guide, with a subsequent quantitative evaluation of the results. The results show an untapped potential on both planning levels. Currently, the greatest success can be seen by various low-regret measures related to urban green.
\end{abstract}


Keywords: Urban land-use planning - Land-use plan . Local development plan - Climate change = Climate change adaptation - Adaptive capacity

\section{Einleitung}

Seit dem Jahr 2008 wird in Deutschland an der Umsetzung der Deutschen Anpassungsstrategie an den Klimawandel (Bundesregierung 2008) und dem 2011 daraus entstandenen Aktionsplan Anpassung (Bundesregierung 2011) gearbeitet. In diesen beiden bundesweit geltenden Strategiepapieren und ihren Fortschrittsberichten werden Städte und Gemeinden als zentrale Akteure zur Bewältigung der Folgen des Klimawandels hervorgehoben (vgl. Bundesregierung 2008: 19; Bundesregierung 2015: 64). Das Ziel ist eine Abmilderung oder gar Abwendung von zunehmenden Klimawandelfolgen wie steigenden Temperaturen, Hitzewellen sowie Starkregenereignissen und deren negativen Konsequenzen für Städte und deren Bevölkerungen. Dieses Ziel kann unter anderem auch durch stadtplanerische Maßnahmen erreicht werden. Bundesministerien, wie das ehemalige Bundesministerium für Verkehr, Bau und Stadtentwicklung (BMVBS 2013a; BMVBS 2013b; BMVBS 2013c) und das Umweltbundesamt (UBA 2016; Albrecht/Schanze/Klimmer et al. 2018), haben im Zuge dessen vermehrt Empfehlungen und Handlungsleitfäden für die Stadt- und Regionalplanung publiziert. Auch die Ministerkonferenz für Raumordnung (MKRO) definierte das Querschnittsthema „Raum-, Regional- und Bauleitplanung sowie Bevölkerungsschutz" bereits im Jahr 2009 zum Handlungserfordernis in Bezug auf den Klimawandel (MKRO 2009).

Die raumplanerischen Zielsetzungen werden, angestoBen durch die Deutsche Anpassungsstrategie an den Klimawandel (Bundesregierung 2008) und den Aktionsplan Anpassung (Bundesregierung 2011), zunächst auf die Landesund regionale Ebene übersetzt, unter anderem durch die Instrumente der Landes- und Regionalplanung. Die weitere Übersetzung auf die kommunale Ebene erfolgt mit den Instrumenten der Bauleitplanung. Diese bildet den Fokus der vorliegenden Untersuchung. Die Grundsätze der Bauleitplanung sind in $\S 1 \mathrm{BauGB}^{1}$ festgehalten und haben die konkrete Aufgabe, zu einer nachhaltigen städtebaulichen Entwicklung beizutragen. Damit setzt die Bauleitplanung den langfristigen planerischen und baulichen Rahmen für gesellschaftliche Bereiche wie Wohnungsbau, Verkehr, Energiewirtschaft, Wasserwirtschaft, Gesundheits-

\footnotetext{
1 Baugesetzbuch in der Fassung der Bekanntmachung vom 3. November 2017, das zuletzt durch Artikel 2 des Gesetzes vom 8. August 2020 geändert worden ist.
}

wesen, Landwirtschaft, Forstwirtschaft, Sicherheit gegenüber Naturgefahren, Naturschutz sowie Versorgung mit Gütern und Dienstleistungen. Durch diese umfassenden Steuerungsmöglichkeiten kann sie strategisch und vorausschauend eingesetzt werden und bei adäquater Integration von Klimaanpassungsmaßnahmen auch zu einer umfassenden Verbesserung der städtischen Anpassungskapazität beitragen (Bundesregierung 2008: 42; Bundesregierung 2015: 55-56). Durch eine Ausschöpfung der bauleitplanerischen Steuerungsmöglichkeiten und Handlungsspielräume können Städte steigenden Temperaturen, Hitzewellen und Starkregenereignissen entgegenwirken.

Zahlreiche deutsche Städte und Gemeinden haben nach der Veröffentlichung der Strategiepapiere dezidierte Klimakoordinierungsstellen eingerichtet, eigene kommunale Klimaanpassungskonzepte und -strategien entwickelt sowie erste entsprechende Maßnahmen auf kommunaler Ebene zur Umsetzung gebracht (vgl. Reckien/Flacke/Dawson et al. 2014: 335; Zimmermann/Boghrat/Weber 2015: 305). Einen erheblichen Einfluss hierauf hatten zum einen bundesweite Förderprogramme, wie das seit dem Jahr 2011 bestehende Förderprogramm zur Deutschen Anpassungsstrategie des Bundesministeriums für Umwelt, Naturschutz und nukleare Sicherheit (BMU) (mit einem Schwerpunkt auf lokalen und regionalen Leuchtturmvorhaben, Anpassungskonzepten für Unternehmen sowie der Entwicklung von Bildungsmodulen) und die Kommunalrichtlinie der Nationalen Klimaschutzinitiative (NKI) zur Förderung von Klimaanpassungs-Teilkonzepten. Darüber hinaus spielen spezielle Förderprogramme einzelner Bundesländer oder Städtebauförderungen entscheidende Rollen (Hasse/Willen/ Baum et al. 2019: 59).

Doch bisher wurde die Auseinandersetzung mit den Folgen des Klimawandels von vielen Gemeinden als eine freiwillige Aufgabe innerhalb ihrer planerischen Tätigkeiten angesehen (Frommer/Schlipf/Böhm et al. 2013: 141). Zahlreiche Gesetzesnovellierungen in den vergangenen Jahren tragen nun jedoch zu einer sukzessiven Stärkung der Themen Klimaschutz und Klimaanpassung bei und betreffen verschiedene Ebenen der räumlichen Planung. Wie auch von Albrecht, Schanze, Klimmer et al. (2018) detailliert zusammengetragen und erörtert, wurden diese Themen sowohl durch klimaanpassungsrelevante Novellierungen des Raumordnungsgesetzes ${ }^{2}$ im Jahr 2008, des Wasserhaushaltsgesetzes ${ }^{3}$ im Jahr 2009 (vgl. Bubeck/Klimmer/Albrecht 2016) und des Gesetzes über die

\footnotetext{
2 Gesetz zur Neufassung des Raumordnungsgesetzes und zur Änderung anderer Vorschriften (GeROG), vom 22. Dezember 2008.

3 Gesetz zur Neuregelung des Wasserrechts vom 31. Juli 2009.
} 
Umweltverträglichkeitsprüfung ${ }^{4}$ im Jahr 2017, aber auch speziell in der rechtlichen Grundlage der Bauleitplanung (Baugesetzbuch) gestärkt. So findet sich im Baugesetzbuch seit der sogenannten Klimaschutznovelle im Jahr 2011 erstmals eine explizite rechtliche Vorgabe zur Förderung von Klimaschutz und Klimaanpassung in der städtebaulichen Entwicklung und innerhalb von Bauleitplänen. Im Jahr 2013 wurde dies durch eine weitere Novelle ergänzt, über die Klimaanpassung nun auch als Aufgabe der städtebaulichen Sanierungs- und Stadtumbauziele definiert wurde. ${ }^{5}$ Die Wichtigkeit einer Regulierung der Flächennutzung in den Gemeinden wird von den Gesetzgebern hierdurch anerkannt. Doch auch wenn die aktuelle rechtliche Grundlage zu einer Stärkung der Klimaanpassung als Abwägungsbelang führt, ist sie keine Garantie für eine tatsächliche Umsetzung innerhalb der bauleitplanerischen Praxis.

Im Rahmen dieses Beitrags soll deshalb der Stand der tatsächlichen Integration von Anpassungsmaßnahmen in aktuellen Bauleitplänen geprüft werden. Dies betrifft sowohl die vorbereitende Bauleitplanung (die Flächennutzungspläne) als auch die verbindliche Bauleitplanung (die Bebauungspläne). Der Flächennutzungsplan stellt das Bindeglied zur überörtlichen Raumordnung dar und gibt die groben Grundzüge der städtebaulichen Entwicklung im gesamten Stadtgebiet vor (Maßstab zwischen 1:10.000 und 1:50.000), während er bezogen auf Grundstücke und deren Eigentümer nicht unmittelbar verbindlich ist. Aus dem Flächennutzungsplan heraus werden in einem anschließenden Schritt Bebauungspläne für kleinräumigere Teilbereiche des Stadtgebiets sowie für konkrete Bau- und Sanierungsvorhaben entwickelt (Maßstab zwischen 1:1.000 und 1:25.000). Sie repräsentieren somit eine parzellenscharfe Planung der kleinräumigen Nutzung und Bebauung und legen rechtsverbindlich die städtebauliche Ordnung fest (vgl. Müller-Ibold 1996: 133-134). Beide Planungsebenen sollen auf die Integration von Anpassungsmaßnahmen geprüft werden.

Die folgende Forschungsfrage leitete die vorliegende Untersuchung: Inwiefern werden Flächennutzungs- und Bebauungspläne als Instrumente zur Integration kommunaler Klimaanpassungsmaßnahmen tatsächlich genutzt (Forschungsfrage 1)?

Im Prozess der Bauleitplanung ist eine gewisse Übersetzungsleistung zwischen beiden Planungsebenen notwendig, welche durch das sogenannte Entwicklungsgebot

\footnotetext{
${ }^{4}$ Gesetz zur Modernisierung des Rechts der Umweltverträglichkeitsprüfung vom 20. Juli 2017.

${ }^{5}$ Weitere Erläuterungen zu den spezifischen rechtlichen Implikationen des Klimaanpassungserfordernisses in der Bauleitplanung werden bei Fleischhauer und Bornefeld (2006), Baumüller (2018) sowie Weidlich (2020) formuliert.
}

abgesteckt wird. Dieses fordert eine Konkretisierung der grobmaschigen Flächennutzungsplanung innerhalb eines Bebauungsplans und dessen kleinräumigeren Planungsgebietes. Flächennutzungspläne werden jedoch in der Regel für einen Zeitraum von 15 bis 20 Jahren aufgestellt (MüllerIbold 1996: 165). Um aufgrund sich ändernder kommunaler Bedarfe allerdings schneller reagieren zu können, ist es umgekehrt auch möglich, in der rechtsverbindlichen Ausgestaltung von Bebauungsplänen bis zu einem gewissen Grad vom übergeordneten Flächennutzungsplan abzuweichen (Rabe/Pauli/Wenzel 2014: 70-71) und letzteren in einem späteren Verfahren anzupassen. Ein Blick auf die Kohärenz klimaanpassungsrelevanter Maßnahmen zwischen beiden Planungsebenen gibt einen Eindruck in die planungshierarchischen Dynamiken innerhalb der Bauleitplanung und über die Rollen beider Planungsebenen als Instrument für die Integration von Klimaanpassung.

Aufgrund dessen geht die vorliegende Untersuchung einer weiteren Forschungsfrage nach: Zeigen sich Flächennutzungspläne und Bebauungspläne innerhalb der gleichen Stadt hinsichtlich ihrer Berücksichtigung von klimaanpassungsrelevanten Maßnahmen als kohärente Instrumente (Forschungsfrage 2)?

Schmitt (2016) untersuchte im Rahmen einer bundesweiten Analyse aller Regionalpläne Deutschlands bereits den Implementationsstand von Klimaanpassungsinhalten auf der regionalen Ebene und identifizierte eine nach wie vor sehr eingeschränkte Integration dieser Inhalte sowie eine meist nur indirekte Einbeziehung von Klimaanpassungsmaßnahmen ohne eine konkrete Begründung durch einen Klimaanpassungsbedarf. Eine durch Buth, Kahlenborn, Savelsberg et al. (2015) durchgeführte Analyse der Vulnerabilität Deutschlands gegenüber dem Klimawandel ermittelte auf der Grundlage einer früheren Studie Schmitts (2014) zu Regionalplänen außerdem eine durchschnittliche Ausschöpfung der Anpassungskapazität von 52 Prozent (Buth/Kahlenborn/Savelsberg et al. 2015: 124-128). Da die übergeordnete Regionalplanung überörtliche Ziele der Raumordnung formuliert, die von der Bauleitplanung zu beachten sind, stellt sich hier die Frage, wie erfolgreich der Integrationsstand von Klimaanpassung auf der nachgeordneten Bauleitplanungsebene noch sein kann, wenn bereits auf der Regionalplanungsebene deutliche Defizite zu verzeichnen sind. Eine Studie von Diepes und Müller (2018) beschrieb aufgrund einer umfassenden Zeitreihenanalyse zwischen 1990 und 2014 die Integration von Klimaanpassungsinhalten in den Bebauungsplänen dreier deutscher Großstädte. Das Ergebnis war auch hier eine defizitäre Integration von Klimaanpassungsmaßnahmen und eine mangelnde Ausschöpfung des bestehenden Handlungsspielraums. Sprondel, Donner und Köppel (2016) unterstreichen mithilfe einer interviewgestützten Wahrscheinlichkeitsbe- 
rechnung am Beispiel Berlins nur limitierte Erfolgswahrscheinlichkeiten klimaanpassungsgerechter Bebauungspläne. Demgegenüber ist eine Studie von Hasse, Willen, Baum et al. (2019) zu nennen, in deren Rahmen Kommunalvertretungen zu den Auswirkungen der Deutschen Anpassungsstrategie an den Klimawandel befragt wurden. Hier gaben die Befragten für das Handlungsfeld „Raum-, Regional- und Bauleitplanung" eine verhältnismäßig hohe Berücksichtigung bzw. Umsetzung von Anpassungsmaßnahmen im Vergleich mit anderen Handlungsfeldern an (Hasse/Willen/Baum et al. 2019: 14, 37). Diese Aussage konnte in den bereits genannten Untersuchungen konkreter Planungsdokumente jedoch nur in limitiertem Maße bestätigt werden.

Ergänzend zu diesem Forschungsstand soll die vorliegende Abhandlung dazu beitragen, den Wissensstand auf die Ebene der Flächennutzungsplanung zu erweitern, welche nach Kenntnis der Autorinnen bisher noch nicht explizit erforscht wurde. Außerdem soll der bisherige Fokus, der auf sehr großen und bevölkerungsreichen Großstädten liegt, um die Perspektive mittelgroßer Städte ergänzt werden. Zusätzlich werden die aktuellen Bauleitpläne - mit Flächennutzungsplänen aus dem Zeitraum 2003 bis 2018 sowie Bebauungsplänen aus dem Zeitraum 2016 bis 2019 herangezogen und repräsentieren somit auch den derzeitigen gesetzlichen Rahmen zum Klimaanpassungserfordernis in Städten und Gemeinden.

Im Folgenden wird zunächst ein Überblick über die Datengrundlage und die Auswahl der Fallstudienstädte und den zugrunde liegenden Bauleitplänen sowie über die methodische Herangehensweise zur Analyse der Pläne gegeben (Kapitel 2). Anschließend erfolgt die Präsentation der Ergebnisse unter Berücksichtigung zentraler klimaanpassungsrelevanter Begriffe, dem Integrationsstand der untersuchten Klimaanpassungsmaßnahmen in den Bauleitplänen sowie der Kohärenz der Bauleitpläne innerhalb der gleichen Stadt (Kapitel 3). Im Rahmen einer Diskussion erfolgt zum Abschluss eine Interpretation und kritische Reflexion der Ergebnisse und methodischen Herangehensweise sowie die Erwähnung weiterer Forschungsbedarfe in diesem Forschungsfeld (Kapitel 4).

\section{Datengrundlage und methodisches Vorgehen}

Das methodische Vorgehen stützt sich auf eine Dokumentenanalyse der Bauleitpläne (Flächennutzungspläne und Bebauungspläne) von zehn deutschen Städten mittlerer Größe und schließt somit große kreisfreie Mittelstädte mit mehr als 50.000 Einwohnern sowie kleine Großstädte mit 100.000 bis 500.000 Einwohnern ein. Städte mittlerer
Größe sind von besonderem Forschungsinteresse, da diese im Vergleich zu Großstädten mit sehr viel limitierteren finanziellen und personellen Ressourcen ausgestattet sind und dementsprechend eher weniger Eigeninitiative in der Umsetzung aufwendiger umweltthematischer Aufgaben ergreifen können (Frommer/Schlipf/Böhm et al. 2013: 141; Bundesregierung 2015: 56). In der Auseinandersetzung mit privaten Planungsträgern und Investoren sind kleinere und mittelgroße Städte in der Ausgestaltung von Planungszielen und Bauvorhaben weniger unabhängig, da sie stärker um die Ansiedelung von Wirtschaftsakteuren werben müssen und sich deren Interessen tendenziell vermehrt unterordnen (Streich 2011: 161-163). In diesem ausgeprägten Spannungsfeld zwischen Klimaanpassungserfordernis einerseits und dem großen Stellenwert anderer städtischer Belange bei gleichzeitiger Ressourcenknappheit andererseits stellt sich die Frage nach der tatsächlichen Integration von Klimaanpassungsmaßnahmen verstärkt.

Neben dem Kriterium der Stadtgröße war auch das Vorhandensein eines bereits öffentlich vorliegenden kommunalen Klimaanpassungskonzepts (KAK) entscheidend für die Fallauswahl. Hierdurch soll sichergestellt werden, dass Städte in die Analyse einfließen, welche bereits eine gewisse Aktivität im Handlungsfeld Klimaanpassung zeigen und entsprechende Maßnahmen demzufolge mit einer höheren Wahrscheinlichkeit innerhalb ihrer Bauleitplanung berücksichtigen. Denn auch wenn informelle Konzepte keine verbindliche Wirkung besitzen, dienen sie doch der gezielten Stärkung von Klimawandelbelangen innerhalb der Stadt und schaffen oft überhaupt erst das Bewusstsein über ein entsprechendes planerisches Erfordernis im Stadtgebiet (Diepes/ Müller 2018: 296). Durch eine Eingrenzung auf diese aktiveren Städte kann die Bandbreite und Ausgestaltung bauleitplanerischer Klimaanpassung gezielter erforscht werden. Trotzdem ist zu beachten, dass die notwendige Bedingung eines vorhandenen kommunalen Klimaanpassungskonzeptes eine mögliche positiv-verzerrende Wirkung auf die Ergebnisse der vorliegenden Untersuchung haben kann. Entsprechend ist eine Übertragung der Ergebnisse auf Städte ohne kommunales Klimaanpassungskonzept nur beschränkt möglich. Eventuell kann man bei diesen Städten eine noch geringere Berücksichtigung von Anpassungsmaßnahmen erwarten.

Bei den zehn Fallstudienstädten handelt es sich um acht kleinere Großstädte (Erfurt, Erlangen, Freiburg im Breisgau, Jena, Karlsruhe, Potsdam, Recklinghausen und Wiesbaden) sowie zwei große kreisfreie Städte (Kaiserslautern und Worms), welche sich auf sieben verschiedene Bundesländer (Bayern, Baden-Württemberg, Brandenburg, Hessen, Rheinland-Pfalz, Thüringen, Nordrhein-Westfalen) verteilen. Konkret befinden sich die Fallstudienstädte damit in einer Größenordnung zwischen etwa 80.000 und 300.000 
Tabelle 1 Veröffentlichungsjahre der untersuchten Bauleitpläne

\begin{tabular}{lllll}
\hline & Kommunales Anpassungskonzept & Flächennutzungsplan & Bebauungsplan 1 & Bebauungsplan 2 \\
\hline Fallstudienstadt 1 & 2018 & 2017 & 2018 & 2019 \\
Fallstudienstadt 2 & 2019 & 2003 & 2016 & 2019 \\
Fallstudienstadt 3 & 2019 & 2006 & 2017 & 2018 \\
Fallstudienstadt 4 & 2012 & 2005 & 2019 & 2019 \\
Fallstudienstadt 5 & 2013 & 2004 & 2018 & 2019 \\
Fallstudienstadt 6 & 2015 & 2013 & 2017 & 2017 \\
Fallstudienstadt 7 & 2017 & 2013 & 2018 & 2019 \\
Fallstudienstadt 8 & 2016 & 2003 & 2018 & 2018 \\
Fallstudienstadt 9 & 2019 & 2018 & 2018 & 2019 \\
Fallstudienstadt 10 & 2016 & 2015 & 2016 & 2016 \\
\hline
\end{tabular}

Einwohnern (Stand 2019). ${ }^{6}$ Eine statistische Repräsentativität für die Gesamtheit mittelgroßer deutscher Städte ist durch diese Studie somit nicht gegeben. Vielmehr sollen allgemeine Tendenzen zum Status der bauleitplanerischen Klimaanpassung beispielhaft anhand der durch die vorhandenen kommunalen Klimaanpassungskonzepte beeinflussten Fallstudienstädte festgestellt werden. Da das Erkenntnisinteresse dieser Untersuchung nicht der Beurteilung einzelner Städte gilt, sondern der Identifikation allgemeiner Dispositionen innerhalb mittelgroßer Städte, werden die untersuchten Fallstudienstädte im Folgenden anonym gehalten, unter anderem durch eine ausschließliche Darstellung von Mittelwerts-Ergebnissen.

Für jede Fallstudienstadt werden sowohl der aktuell gültige Flächennutzungsplan (insgesamt zehn Pläne) als auch zwei exemplarisch ausgewählte Bebauungspläne analysiert (insgesamt 20 Pläne). Alle Bebauungspläne sind aktueller als der jeweils übergeordnete Flächennutzungsplan, um eine inhaltliche Kohärenz zwischen beiden Planungsebenen (vgl. Forschungsfrage 2) überhaupt prüfen zu können. Außerdem repräsentieren sie die aktuellsten auswertbaren Bebauungspläne der Fallstudienstädte. Als nicht auswertbar gelten Pläne, welche lediglich einen Nutzungsausschluss festsetzen oder sehr geringfügige Änderungen zur bereits bestehenden Planung aufweisen. Hieraus ergibt sich auch, dass einige untersuchte Bebauungspläne als (fast abgeschlossene) Entwurfsversionen vorlagen, da es zum Zeitpunkt der Untersuchung nur wenige, kürzlich rechtsverbindlich veröffentlichte Bebauungspläne gab. In solchen Fällen wird davon ausgegangen, dass sich der Planinhalt zum Zeitpunkt der rechtswirksamen Veröffentlichung höchstens geringfügig verändern wird. Alle untersuchten Bebauungspläne wurden außerdem nach den BauGB-Novellen der Jahre 2011 und 2013

\footnotetext{
${ }^{6}$ Statistisches Bundesamt 2020: https://www.destatis.de/DE/ Themen/Laender-Regionen/Regionales/Gemeindeverzeichnis/ Administrativ/05-staedte.html/ (09.04.2021).
}

veröffentlicht. Somit fließen nur solche Pläne in die Untersuchung ein, welche einem nun auch gesetzlich verankerten Klimaanpassungs-Leitbild folgen sollen. Darüber hinaus wurde bei der Auswahl der Bebauungspläne darauf geachtet, dass sie möglichst vielfältiger Art sind. So wurden neben nutzungsbezogenen Änderungen (z. B. die Umnutzung eines vorherigen Gewerbegebietes in ein Wohngebiet) auch vorhabenbezogene Bebauungspläne (z. B. Bauvorhaben durch einen Vorhabenträger bzw. Investor) untersucht, sowie möglichst diverse geographische Lagen (z. B. Innenstadtnähe oder Außenbereich) und Nutzungsarten (z. B. Gewerbegebiet, Wohngebiet, Sondergebiet) abgedeckt. Hierdurch sollen möglichst vielfältige Bauleitplanungsverfahren und Kontexte widergespiegelt werden.

Die Veröffentlichungsjahre der untersuchten Pläne sind in Tabelle 1 aufgeführt. Diese Flächennutzungspläne waren zwischen 2003 und 2018 veröffentlicht worden, während die Bebauungspläne im Zeitraum zwischen 2016 und 2019 liegen. Aus Tabelle 1 wird darüber hinaus ersichtlich, dass die untersuchten Flächennutzungspläne allesamt bereits vor der Veröffentlichung des jeweiligen kommunalen Anpassungskonzeptes rechtswirksam wurden. Dies liegt darin begründet, dass Flächennutzungspläne nur in relativ großen zeitlichen Abständen neu aufgestellt werden und entsprechend häufig bereits schon seit vielen Jahren bestehen. Im Gegensatz dazu ist der Großteil aller untersuchten Bebauungspläne erst nach der Veröffentlichung des jeweiligen kommunalen Anpassungskonzeptes in Kraft getreten. Der Einfluss informeller Konzepte auf die Integration klimaanpassungsrelevanter Maßnahmen in der Bauleitplanung ist mit Hinblick auf die vorliegende Datengrundlage also nur auf Bebauungsplanebene abschätzbar.

In die Untersuchung fließen neben Planzeichnungen auch die zugehörigen textlichen Planungsdokumente wie textliche Festsetzungen, Begründungen und Umweltberichte ein, sofern diese vorlagen. Mit insgesamt 25 untersuchten Planungsdokumenten auf der Ebene der Flächennutzungsplanung und 56 untersuchten Dokumenten der Bebauungspla- 
Tabelle 2 Übersicht über alle untersuchten Handlungsfelder und Klimaanpassungsmaßnahmen

\begin{tabular}{|c|c|c|c|c|c|}
\hline \multirow{2}{*}{$\begin{array}{l}\text { Handlungsfeld } \\
\text { Bauwesen }\end{array}$} & \multicolumn{5}{|c|}{ Anpassungsmaßnahmen } \\
\hline & $\begin{array}{l}\text { Gebäudeschutz ge- } \\
\text { gen Hitze }\end{array}$ & $\begin{array}{l}\text { Gebäudeschutz ge- } \\
\text { gen Starkregen und } \\
\text { Hochwasser }\end{array}$ & Gebäudebegrünung & $\begin{array}{l}\text { Kaltluft- und } \\
\text { Frischluftareale }\end{array}$ & \\
\hline $\begin{array}{l}\text { Grün- und Freiflä- } \\
\text { chen }\end{array}$ & $\begin{array}{l}\text { Sicherung grüner } \\
\text { Infrastruktur }\end{array}$ & $\begin{array}{l}\text { Neuschaffung grüner } \\
\text { Infrastruktur }\end{array}$ & Freiraumvernetzung & $\begin{array}{l}\text { Mikroklimatisches } \\
\text { Stadtgrün }\end{array}$ & \\
\hline Infrastruktur & $\begin{array}{l}\text { Kanalisations- } \\
\text { optimierung }\end{array}$ & $\begin{array}{l}\text { Hitzeanpassung von } \\
\text { Straßenbelägen }\end{array}$ & & & \\
\hline $\begin{array}{l}\text { Biodiversität und } \\
\text { Naturschutz }\end{array}$ & $\begin{array}{l}\text { Schutz für Moore, } \\
\text { Feuchtgebiete und } \\
\text { Auen* }\end{array}$ & $\begin{array}{l}\text { Schutz für Oberflä- } \\
\text { chengewässer* }\end{array}$ & $\begin{array}{l}\text { Unterstützung von } \\
\text { Biotopverbundsys- } \\
\text { temen* }\end{array}$ & $\begin{array}{l}\text { Neobiota-Ma- } \\
\text { nagement }\end{array}$ & \\
\hline Wasserwirtschaft & $\begin{array}{l}\text { Natürliches Regen- } \\
\text { wassermanagement }\end{array}$ & $\begin{array}{l}\text { Technisches Regen- } \\
\text { wassermanagement }\end{array}$ & $\begin{array}{l}\text { Vor-Ort-Entwässe- } \\
\text { rung }\end{array}$ & $\begin{array}{l}\text { Grundwasser- } \\
\text { management }\end{array}$ & $\begin{array}{l}\text { Ausweitung der } \\
\text { Trinkwasserver- } \\
\text { sorgung }\end{array}$ \\
\hline $\begin{array}{l}\text { Risikovorsorge } \\
\text { bei Naturkata- } \\
\text { strophen }\end{array}$ & $\begin{array}{l}\text { Natürlicher Hoch- } \\
\text { wasserschutz* }\end{array}$ & $\begin{array}{l}\text { Technischer Hochwas- } \\
\text { serschutz* }^{*}\end{array}$ & $\begin{array}{l}\text { Starkregen-Manage- } \\
\text { ment }\end{array}$ & $\begin{array}{l}\text { Bodenerosions- } \\
\text { management }\end{array}$ & $\begin{array}{l}\text { Risikogebiets- } \\
\text { Monitoring }\end{array}$ \\
\hline
\end{tabular}

*Diese Anpassungsmaßnahmen werden aufgrund fehlender räumlicher Gegebenheiten im lokalen Planungsgebiet auf der Bebauungsplan-Ebene nicht untersucht.

nung-Ebene umfasst die vorliegende Untersuchung somit eine Gesamtheit von 81 Planungsdokumenten.

Die Untersuchung stützt sich auf eine qualitative strukturierende Inhaltsanalyse der genannten Bauleitplanungsdokumente, geleitet durch einen vorab entwickelten, kategoriengestützten Kodierleitfaden (vgl. Mayring/Fenzl 2014: 548). Die Ergebnisse wurden im Nachgang weiterhin quantitativ ausgewertet. Das Verfahren hat den Vorteil, durch die Bildung von Kategorien regelgeleitete und am Erkenntnisinteresse der Forschungsfragen ausgerichtete und darauf beschränkte Informationen aus den zugrunde liegenden Daten zu extrahieren. Ein Nachteil der Methode besteht darin, dass falsch gebildete Kategorien zu einer Verzerrung der Zusammenhänge führen würden und mögliche ,blinde Flecken' nicht erfasst werden können. Diesem Risiko wurde durch die Verwendung einschlägiger Literatur sowie einem iterativen Prozess der Kategorienbildung begegnet. Dieses Vorgehen wird im Folgenden näher erläutert.

Neben der inhaltlichen Auseinandersetzung mit konkreten Maßnahmen erfolgt zunächst eine Kodierung von allgemein klimaanpassungsrelevanten Begriffen wie Klimawandel, Klimaanpassung, Hitze und Starkregen, um den Stellenwert dieser Themen als städtische Belange und die Rolle von Klimaanpassung in der konzeptionellen Ausrichtung der untersuchten Bauleitpläne zu ermitteln. Das Ausmaß der Integration solcher Begriffe in Planungsdokumenten kann als Hinweis gewertet werden und die Frage aufwerfen, ob die untersuchten Maßnahmen tendenziell eher durch eine Klimaanpassungsmotivation oder stattdessen durch andere Interessen begründet sind. Jeder dieser Begriffe wird anhand vielfacher möglicher Suchworte geprüft (z. B. Hit- ze: Überhitzung, Hitzeperioden, Hitzespitzen, Wärmebelastung, Wärmeinsel, Extremtemperatur).

Für die weiterführende Untersuchung wird ein theoriegeleitetes Kategoriensystem aus zahlreichen Klimaanpassungsmaßnahmen des bauleitplanerischen Kontexts identifiziert. Dem System liegen mehrere Quellen zugrunde: die Deutsche Anpassungsstrategie an den Klimawandel (DAS; Bundesregierung 2008), der Aktionsplan Anpassung (Bundesregierung 2011), der Festsetzungskatalog nach $\S 9$ BauGB sowie die „Planungsbezogenen Empfehlungen zur Klimaanpassung auf Basis des Stadtklimalotsen“ (BMVBS 2013b) und die „Praxishilfe - Klimaanpassung in der räumlichen Planung“ (UBA 2016). Anhand dieser Vorgaben wurden für die vorliegende Untersuchung 24 verschiedene Klimaanpassungsmaßnahmen mit Bezug zur Bauleitplanung übernommen, welche wiederum in sechs verschiedene Handlungsfeld-Kategorien unterteilt werden: Bauwesen, Grün- und Freiflächen, Infrastruktur, Biodiversität und Naturschutz, Wasserwirtschaft sowie Risikovorsorge bei Naturkatastrophen (vgl. Tabelle 2). Auf der Ebene der Flächennutzungspläne wurden alle 24 Maßnahmen untersucht, während auf der Bebauungsplan-Ebene nur 19 Maßnahmen geprüft wurden, da die übrigen fünf Maßnahmen auf dieser Ebene nicht geprüft werden können. Dies liegt in den zugrunde liegenden räumlichen Gegebenheiten des lokalen Planungsgebiets auf der Ebene des Bebauungsplans begründet, welche großteils keine Umsetzung der jeweiligen Maßnahmen zulassen (z. B. das Fehlen von Gewässern lässt keine Klimaanpassung im Bereich Hochwasserschutz zu). Entsprechend wurden die fünf betroffenen Maßnahmen lediglich auf der Ebene der Flächennutzungspläne untersucht, jedoch nicht auf der Ebene der Bebauungspläne. 
Im weiteren Verlauf gilt zu beachten, dass die Gesamtzahl der erreichbaren Klimaanpassungsmaßnahmen in dieser Untersuchung jeweils normalisiert wurde, das heißt auf der Ebene der Flächennutzungspläne entsprechen $24 \mathrm{Maßnah-}$ men, auf der Ebene der Bebauungspläne 19 Maßnahmen den 100 Prozent erreichbarer Maßnahmen.

Das so entstandene theoriegeleitete Kategoriensystem bildet aggregierte Anpassungsmaßnahmen-Kategorien ab. Jede der 24 Anpassungsmaßnahmen fußt wiederum auf zahlreichen untergeordneten Suchworten, welche auf der bereits genannten Literatur fundieren und zur Kodierung der Bauleitplanungsdokumente herangezogen wurden. Diese Suchworte werden zu Beginn anhand eines Trainingsdatensatzes in einem iterativen Prozess angewandt und angepasst, um die terminologischen Ausprägungen dieser speziellen Planungsdokumente zu treffen. Jedes dieser Suchworte kann als eigenständige Klimaanpassungsmaßnahme verstanden werden, jedoch wurden diese im Rahmen der vorliegenden Untersuchung aus Gründen der Vereinfachung in die bereits genannten 24 aggregierten Anpassungsmaßnahmen überführt. Beispielsweise wurde die Anpassungsmaßnahme ,natürliches Regenwassermanagement" anhand folgender Suchworte in den Planungsdokumenten kodiert: begrünte Mulden, Gräben oder Rinnen; Entsiegelung oder Versiegelungsvermeidung; wasserdurchlässige Bodengestaltung; Gebäudebegrünung. Tatsächlich wurde also eine sehr umfassende Anzahl verschiedener Anpassungsmaßnahmen geprüft. Eine vollständige Auflistung des kategoriengestützten Kodierleitfadens mitsamt aller Handlungsfelder, Klimaanpassungsmaßnahmen und Suchworte ist Tabelle $3 \mathrm{zu}$ entnehmen. Hier wird auch deutlich, dass in wenigen Fällen eine Doppelkodierung von Maßnahmen stattfand, sofern den Planungsdokumenten klar zu entnehmen war, dass die Maßnahme durch mehrere Motivationen begründet wird (entsprechende Suchworte sind in Tabelle 3 mit Sternchen markiert). Im Fall der Gebäudebegrünung liegt diese beispielsweise als eigene Anpassungsmaßnahme vor, allerdings auch als Suchwort bei weiteren Anpassungsmaßnahmen, wie in diesem Fall bei „mikroklimatischem Stadtgrün“ oder „,natürlichem Regenwassermanagement“. Solche Überschneidungen sind aufgrund des multifunktionalen Charakters von klimaanpassungsrelevanten Maßnahmen nicht auszuschließen.

Sofern in den untersuchten Planungsdokumenten eine klare Berücksichtigung dieser Anpassungsmaßnahmen stattfand, wurden die jeweiligen Textstellen dem Kodierleitfaden entsprechend eingeordnet und deren Integrationsstand als „positiv" bewertet. $\mathrm{Zu}$ beachten ist, dass eine solche Bewertung als ,positiv“ bereits bei Vorhandensein eines einzigen passenden Suchwortes vorgenommen wurde. Das heißt, die Anzahl der im Plandokument berücksichtigten untergeordneten Suchworte hat keine weitere Auswirkung auf die
„positiv“-Bewertung der Anpassungsmaßnahme und es gibt keinen Schwellenwert, ab welchem erst eine Bewertung als „positiv“ gekennzeichnet wird (Beispiel Anpassungsmaßnahme „Gebäudeschutz gegen Hitze“: Wurde das Suchwort „optimale Gebäudestellung“" gefunden, so wurde die Anpassungsmaßnahme als positiv bewertet; Selbiges ist der Fall, wenn zusätzlich auch die Suchworte ,helle Fassaden und Dächer“ und/oder ,gezielte Verschattung“ verwendet wurden (vgl. Tabelle 3)). Im Falle einer fehlenden Berücksichtigung wurde die Maßnahme als „negativ“ bewertet. Diese Analyse mündet in die Beantwortung von Forschungsfrage 1 .

Zur Beantwortung von Forschungsfrage 2 wird in einem nächsten Schritt die Kohärenz zwischen beiden Bauleitplanungsebenen innerhalb der gleichen Fallstudienstadt untersucht. Hierfür wird geprüft, ob die kodierten Klimaanpassungsmaßnahmen der Bebauungspläne mit denen der jeweilig übergeordneten Flächennutzungspläne übereinstimmen oder nicht. Dabei werden vier verschiedene Kohärenz-Fälle unterschieden. Zum einen können die beiden Planungsebenen kohärent sein: Positiv kohärente Maßnahmen liegen vor, wenn die untersuchte Maßnahme sowohl im Flächennutzungsplan als auch im Bebauungsplan integriert ist. Negativ kohärente Maßnahmen liegen vor, wenn diese auf beiden Planungsebenen gleichzeitig fehlen. Zum anderen können die beiden Planungsebenen inkohärent sein: Inkohärent (Flächennutzungsplan als Vorreiter) bedeutet, dass die Maßnahme ausschließlich auf der Ebene des Flächennutzungsplanes integriert ist, auf der Ebene des Bebauungsplanes jedoch nicht. Der gegenteilige Fall wird entsprechend als inkohärent (Bebauungsplan als Vorreiter) bezeichnet.

Alle diese auf einer qualitativen strukturierenden Inhaltsanalyse basierenden Kodierungen werden im Anschluss in numerische Werte überführt und anhand statistisch ermittelter Prozentanteile bzw. Häufigkeitsverteilungen quantitativ ausgewertet und anschließend aggregiert graphisch visualisiert. Hierdurch soll ein Gesamteindruck über die Fallstudienstädte hinweg ermittelt werden, um Muster innerhalb der bauleitplanerischen Klimaanpassung zu erkennen, ohne dabei die individuellen Fallstudienstädte zu beurteilen.

\section{Ergebnisse}

Die Ergebnisse dieser Untersuchung werden anhand von Häufigkeitsverteilungen dargestellt und interpretiert. Neben der Nennung klimaanpassungsrelevanter Begriffe werden auch die Integration klimaanpassungsrelevanter Maßnahmen innerhalb der untersuchten Flächennutzungspläne und Bebauungspläne sowie die inhaltliche Kohärenz zwischen Flächennutzungsplänen und Bebauungsplänen behandelt. Letztere Ergebnisse werden sowohl im Mittel als auch 
Tabelle 3 Übersicht über die untersuchten Klimaanpassungsmaßnahmen (linke Spalte) pro Handlungsfeld und entsprechende Suchworte (rechte Spalte)

\begin{tabular}{|c|c|}
\hline Klimaanpassungsmaßnahme & Suchworte \\
\hline \multicolumn{2}{|l|}{ Handlungsfeld: Bauwesen } \\
\hline Gebäudeschutz gegen Hitze & $\begin{array}{l}\text { - Optimale Gebäudestellung } \\
\text { - Helle Fassaden und Dächer } \\
\text { - Energetische Optimierung/Sanierung } \\
\text { - Gezielte Verschattung } \\
\text { - Angepasste Baumaterialien (z. B. zur Temperatur-Amplitudendämpfung oder massivere } \\
\text { Materialien an Innenschicht von Gebäuden) } \\
\text { - Dämmmaterialien (an der Außenseite von Gebäuden) } \\
\text { - Vermeidung von Glasarchitektur und Glasfassaden } \\
\text { - Kühlung, Klimatisierung oder Klimaanlagen } \\
\text { - Dachbegrünung, Fassadenbegrünung oder Wandbegrünung* } \\
\text { - Gebäudenahe Grünflächen* }\end{array}$ \\
\hline $\begin{array}{l}\text { Gebäudeschutz gegen Starkregen und } \\
\text { Hochwasser }\end{array}$ & $\begin{array}{l}\text { - Festsetzung von Fußbodenoberkante oder Straßenoberkante } \\
\text { - Wasserdichter Gebäudebau: } \\
\text { - z. B. Kellerfenster, Fenster und Türen, Wanne, Weiße Wanne, Frostschutzschürze } \\
\text { - z. B. wasserdichte Bodenbeläge: kapillarbrechende Schicht, Kiessand oder Schotter } \\
\text { - z. B. Verzicht auf Unterkellerung } \\
\text { - z. B. absperrbarer Kanalanschluss } \\
\text { - Sicherung von Gebäuden gegen Aufschwimmen } \\
\text { - Flutbare Tiefgaragen } \\
\text { - Sicherheitsrelevante technische Anlagen erhöht anbringen } \\
\text { - Mobile Anlagen }\end{array}$ \\
\hline Gebäudebegrünung & $\begin{array}{l}\text { - Dachbegrünung } \\
\text { - Fassadenbegrünung } \\
\text { - Wandbegrünung }\end{array}$ \\
\hline Kaltluft- und Frischluftareale & $\begin{array}{l}\text { - Freihaltung oder Schaffung von Frischluftgebieten, Kaltluftgebieten und Luftleitbahnen } \\
\text { - Freihaltung oder Schaffung von klimarelevanten Schutzzonen } \\
\text { - Vermeidung von Aufforstungen } \\
\text { - Regionale Grünzüge } \\
\text { - Innerstädtische Grünflächen* } \\
\text { - Grünverbindungen oder Grünzäsuren* }\end{array}$ \\
\hline \multicolumn{2}{|l|}{ Handlungsfeld: Grün- und Freiflächen } \\
\hline Sicherung grüner Infrastruktur & $\begin{array}{l}\text { - Sicherung, Erhalt und Pflege von bestehender städtischer Vegetation } \\
\text { (flächige, linienhafte sowie punktuelle Vegetation) } \\
\text { - Ersatz von bestehender Stadtvegetation im Falle von Verlusten } \\
\text { - Förderung klimaangepasster Arten }\end{array}$ \\
\hline Neuschaffung grüner Infrastruktur & $\begin{array}{l}\text { - Pflanzung und Anlegen von neuer städtischer Vegetation } \\
\text { (flächige, linienhafte sowie punktuelle Vegetation) }\end{array}$ \\
\hline Freiraumvernetzung & $\begin{array}{l}\text { - Vernetzung von Offenflächen, Grünflächen, Grünfingern, Grünzäsuren } \\
\text { - Anlegen von Freiraumachsen }\end{array}$ \\
\hline Mikroklimatisches Stadtgrün & $\begin{array}{l}\text { Alle Maßnahmen der grünen Infrastruktur mit klarer Motivation durch deren begrenzt mikro- } \\
\text { klimatische Wirksamkeit: } \\
\text { - Dachbegrünung, Fassadenbegrünung oder Wandbegrünung* } \\
\text { - Sicherung, Erhalt und Pflege von bestehender städtischer Vegetation (flächige, linienhafte } \\
\text { sowie punktuelle Vegetation)* } \\
\text { - Pflanzung und Anlegen von neuer städtischer Vegetation (flächige, linienhafte sowie punk- } \\
\text { tuelle Vegetation)* }\end{array}$ \\
\hline
\end{tabular}

aggregiert nach den sechs Handlungsfeldern und als Einzelmaßnahmen betrachtet. Bei der Mittelwertberechnung handelt es sich stets um das arithmetische Mittel.

\subsection{Untersuchung klimaanpassungsrelevan- ter Begriffe}

Zunächst wird analysiert, mit welcher Häufigkeit die vier Begriffe Klimawandel, Klimaanpassung, Hitze und Starkregen innerhalb der untersuchten Bauleitpläne vorkommen. Hierzu wurden pro Begriff jeweils die Häufigkeiten der 
Tabelle 3 (Fortsetzung)

\begin{tabular}{|c|c|}
\hline Klimaanpassungsmaßnahme & Suchworte \\
\hline \multicolumn{2}{|l|}{ Handlungsfeld: Infrastruktur } \\
\hline Kanalisationsoptimierung & $\begin{array}{l}\text { - Vermeidung von Abflussspitzen in der Kanalisation } \\
\text { - Trennkanalisation } \\
\text { - Sicherung von Räumen gegen Rückstau von Abwasser } \\
\text { - Beachtung und Anpassung von Rückstauebenen } \\
\text { - Rückstausicherungen in Abwasserleitungen }\end{array}$ \\
\hline Hitzeanpassung von Straßenbelägen & $\begin{array}{l}\text { - Hitzebeständiger Straßenbelag } \\
\text { - Hitzeresistenter Asphalt (z. B. polymer-modifiziert) } \\
\text { - Verwendung heller Materialien (Erhöhung der Albedo) }\end{array}$ \\
\hline \multicolumn{2}{|c|}{ Handlungsfeld: Biodiversität und Naturschutz } \\
\hline $\begin{array}{l}\text { Schutz für Moore, Feuchtgebiete und } \\
\text { Auen }\end{array}$ & $\begin{array}{l}\text { - Schutz und Renaturierung von Mooren, Feuchtgebieten } \\
\text { - Schutz und Renaturierung von Auen, Auenrevitalisierung }\end{array}$ \\
\hline Schutz für Oberflächengewässer & $\begin{array}{l}\text { - Verbesserung des hydromorphologischen Zustands von Gewässern } \\
\text { - Verbesserung der linearen Durchgängigkeit von Fließgewässern } \\
\text { - Renaturierung von Gewässern } \\
\text { - Vermeidung und Verhinderung von Nährstoffeinträgen } \\
\text { - Anpflanzungen an Gewässern }\end{array}$ \\
\hline $\begin{array}{l}\text { Unterstützung von Biotopverbundsys- } \\
\text { temen }\end{array}$ & $\begin{array}{l}\text { - Schutz und Förderung von Biotopverbundsystemen } \\
\text { - Vernetzung von Biotopen }\end{array}$ \\
\hline Neobiota-Management & - Entfernung gebietsfremder und invasiver Arten, Alien Species \\
\hline \multicolumn{2}{|l|}{ Handlungsfeld: Wasserwirtschaft } \\
\hline Natürliches Regenwassermanagement & $\begin{array}{l}\text { - Anlegen von Mulden, Gräben, Rinnen (meist begrünt) } \\
\text { - Entsiegelung oder Versiegelungsvermeidung } \\
\text { - Wasserdurchlässige Bodenbeläge (z. B. Pflaster mit breiten Zwischenfugen, Schotter, Kies) } \\
\text { - Dachbegrünung, Fassadenbegrünung oder Wandbegrünung* }\end{array}$ \\
\hline Technisches Regenwassermanagement & $\begin{array}{l}\text { - Rigolen } \\
\text { - Schächte } \\
\text { - Zisternen } \\
\text { - Entwässerungskanäle } \\
\text { - Straßenabläufe, Gullys } \\
\text { - Regenrückhaltebecken, Regenüberlaufbecken } \\
\text { - Regenklärwerke } \\
\text { - Querneigung der Straße } \\
\text { - NASS („Neuartige Sanitärsysteme“) } \\
\text { - Sickergalerien } \\
\text { - Tiefstollen, Flachstollen } \\
\text { - Wasserdurchlässiger Asphalt, offenporiger Asphalt }\end{array}$ \\
\hline Vor-Ort-Entwässerung & - Dezentrale Regenwasserversickerung/-einleitung auf Grundstücken \\
\hline Grundwassermanagement & $\begin{array}{l}\text { - Förderung der Grundwasserneubildung (z. B. durch Förderung der Wasserversickerung) } \\
\text { - Balancierte Grundwasserentnahme } \\
\text { - Monitoring von Grundwasserständen* } \\
\text { - Gebot zur Nutzung bestehender Grundwasservorkommen anstatt Erschließung neuer } \\
\text { - Prävention/Reduzierung von Nährstoffeinträgen in das Grundwasser }\end{array}$ \\
\hline Ausweitung der Trinkwasserversorgung & $\begin{array}{l}\text { - Dezentralisierung der Trinkwasserversorgung } \\
\text { - Projektion/Prognose künftigen Wasserdargebots, strategische Planung öffentlicher Was- } \\
\text { serversorgung } \\
\text { - Erhöhung des Anschlussgrades } \\
\text { - Erhöhung von Gruppenwasserversorgungen } \\
\text { - Erhöhung des Fernwasseranteils/überregionale Versorgungssysteme (interkommunale } \\
\text { Zusammenarbeit) }\end{array}$ \\
\hline
\end{tabular}

zehn Flächennutzungspläne (blau) sowie der 20 Bebauungspläne (rot) gemittelt. Dabei ist deutlich zu erkennen, dass alle untersuchten Begriffe nur mit eingeschränkter Häufigkeit genannt werden. Außerdem zeigt sich, dass die Mehrheit der Begriffe verstärkt in Flächennutzungsplänen enthalten sind, nämlich in den Fällen Klimawandel, Hitze und Starkregen. Dabei wird Klimawandel in 50\% aller Flächennutzungspläne erwähnt, jedoch in nur 30\% aller Bebauungspläne. Ein Hitze-Bezug wird sogar in $70 \%$ der Flächennutzungspläne, aber in nur $30 \%$ der Bebauungspläne hergestellt. An 
Tabelle 3 (Fortsetzung)

\begin{tabular}{|c|c|}
\hline Klimaanpassungsmaßnahme & Suchworte \\
\hline \multicolumn{2}{|c|}{ Handlungsfeld: Risikovorsorge bei Naturkatastrophen } \\
\hline Natürlicher Hochwasserschutz & $\begin{array}{l}\text { - Sicherung natürlicher Überschwemmungsgebiete: } \\
\text { - Sicherung von Überflutungsauen, Grünland und Freiräumen } \\
\text { - Vermeidung bzw. Verbot von Bebauung } \\
\text { - Entsiegelung oder Versiegelungsvermeidung zur Versickerungsförderung }\end{array}$ \\
\hline Technischer Hochwasserschutz & $\begin{array}{l}\text { - Hochwasserschutzanlagen: } \\
\text { - Deiche, Sperrwerke oder Dünen } \\
\text { - Talsperren } \\
\text { - Hochwasser-Rückhaltebecken } \\
\text { - Hochwasser-Polder } \\
\text { - Hochwasser-Zisternen } \\
\text { - Versickerungsanlagen oder Rigolen zum Hochwasserschutz }\end{array}$ \\
\hline Starkregen-Management & $\begin{array}{l}\text { Kategorie ist nur positiv bewertbar, wenn zentraler Begriff „Starkregen“ in Plänen vor- } \\
\text { kommt. } \\
\text { - Alle Regenwassermanagement-Maßnahmen mit klarer Motivation zum Starkregen-Manage- } \\
\text { ment } \\
\text { - Notwasserwege }\end{array}$ \\
\hline Bodenerosions-Management & $\begin{array}{l}\text { - Erosionsabwehr durch pflanzenbauliche Maßnahmen } \\
\text { - Erosionsabwehr durch Erhaltung von Strukturelementen der Feldflur (z. B. Hecken, Feldge- } \\
\text { hölze, Acker-Terrassen) } \\
\text { - Berücksichtigung der Hangneigung sowie Wasser-/Windverhältnisse } \\
\text { - Nachhaltige Weidepraktiken } \\
\text { - Monitoring erodierender Flächen }\end{array}$ \\
\hline
\end{tabular}

*Diese Anpassungsmaßnahmen-Suchworte sind von einer Doppelkodierung betroffen (das heißt, sie werden zur Bewertung von mehr als einer Anpassungsmaßnahme herangezogen).

dieser Stelle sei erwähnt, dass Bebauungspläne des Innenstadtbereichs (welche tendenziell vermehrt von Hitzephänomenen betroffen sind) nicht nennenswert mehr oder weniger Hitze-Bezug aufweisen als Bebauungspläne außerhalb der Innenstädte. Ein Starkregen-Bezug kommt nur in 50\% der Flächennutzungspläne und in $40 \%$ der Bebauungspläne vor. Der Begriff Klimaanpassung wird auf beiden Bauleitplanungsebenen gleichermaßen mit einer 40-Prozent-Quote genannt.

\subsection{Untersuchung der Integration von klimaanpassungsrelevanten Maßnahmen in Flächennutzungs- und Bebauungsplänen}

Nachfolgend wird gezeigt, mit welcher Häufigkeit die Gesamtheit aller untersuchten Klimaanpassungsmaßnahmen im Durchschnitt aufgeführt werden. Auf der Ebene der Flächennutzungspläne gehen alle $24 \mathrm{Maßnahmen} \mathrm{in} \mathrm{den}$ Mittelwert ein, bei den Bebauungsplänen sind es lediglich 19 prüfbare Maßnahmen (normalisiert) (vgl. Tabelle 2). Ein Blick auf die aggregierte Häufigkeit über alle Maßnahmen und Handlungsfelder gemittelt macht deutlich, dass beide Bauleitplanungsebenen derzeit nur in eingeschränktem Maße klimaanpassungsrelevante Maßnahmen integrieren, wobei sich beide Ebenen jedoch auch deutlich unterscheiden. Während durchschnittlich noch $56 \%$ aller untersuchten
Maßnahmen in den Flächennutzungsplänen enthalten sind, sind es bei den Bebauungsplänen nur noch 40 Prozent. Dieser allgemeine Trend kann nach einer Aufschlüsselung der verschiedenen Handlungsfelder sowie der einzelnen Maßnahmen differenzierter betrachtet werden.

Abbildung 1 zeigt, mit welcher Häufigkeit die Anpassungsmaßnahmen, unterteilt nach den sechs verschiedenen Handlungsfeldern, in Bauleitplänen integriert werden. Dabei wird zunächst für jeden untersuchten Plan ermittelt, wie viel Prozent der erreichbaren Maßnahmen innerhalb eines Handlungsfeldes tatsächlich berücksichtigt sind. Wurden in einem Plan beispielsweise drei von vier Maßnahmen im Feld Bauwesen benannt, so wurde eine prozentuale Häufigkeit von $75 \%$ der erreichbaren Maßnahmen festgestellt. Im Anschluss daran wird für alle Flächennutzungspläne sowie für alle Bebauungspläne ein arithmetischer Mittelwert der vorliegenden prozentualen Häufigkeiten pro Handlungsfeld gebildet, wie er in Abbildung 1 dargestellt ist. Hier sei erneut darauf hingewiesen, dass bei der Untersuchung von Bebauungsplänen weniger Maßnahmen in den Handlungsfeldern Biodiversität und Naturschutz sowie Risikovorsorge bei Naturkatastrophen (siehe rot schraffierte Balken) erkannt wurden als bei den Flächennutzungsplänen. Als Ergebnis ist zunächst festzuhalten, dass in fünf von sechs Handlungsfeldern deutlich mehr Maßnahmen auf der Ebene der Flächennutzungspläne notiert sind, als dies auf der Ebene der Bebauungspläne der Fall ist. Hiervon betroffen sind die Hand- 


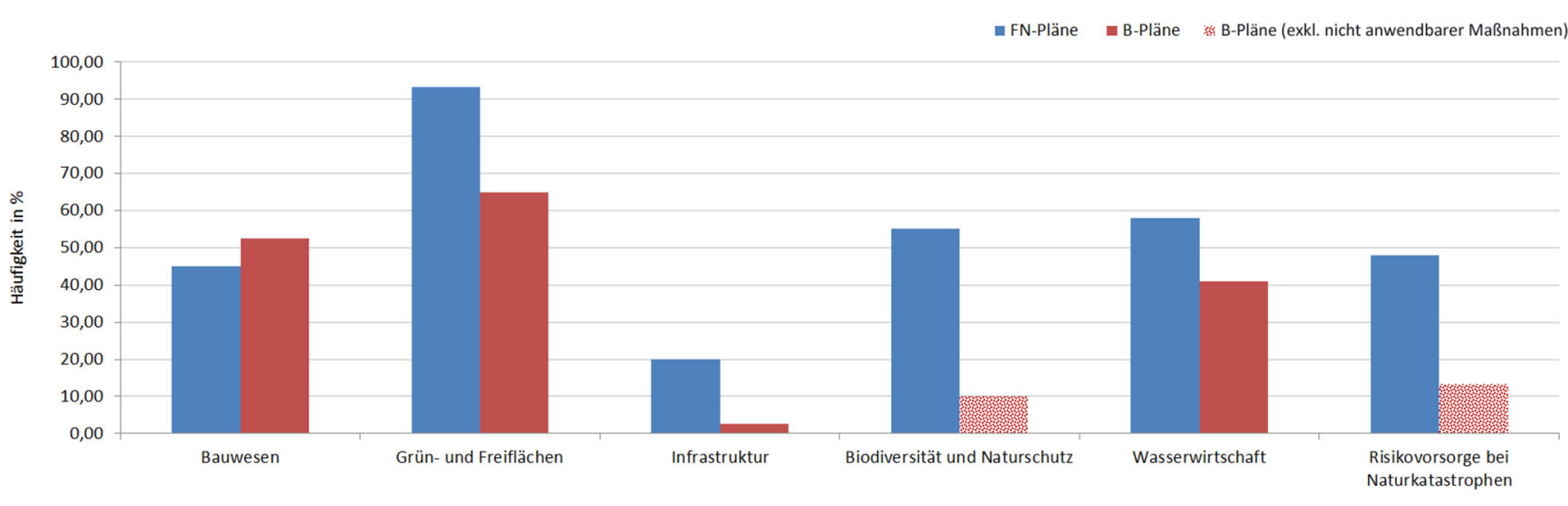

Abbildung 1 Durchschnittliche Häufigkeit der untersuchten Anpassungsmaßnahmen pro Handlungsfeld

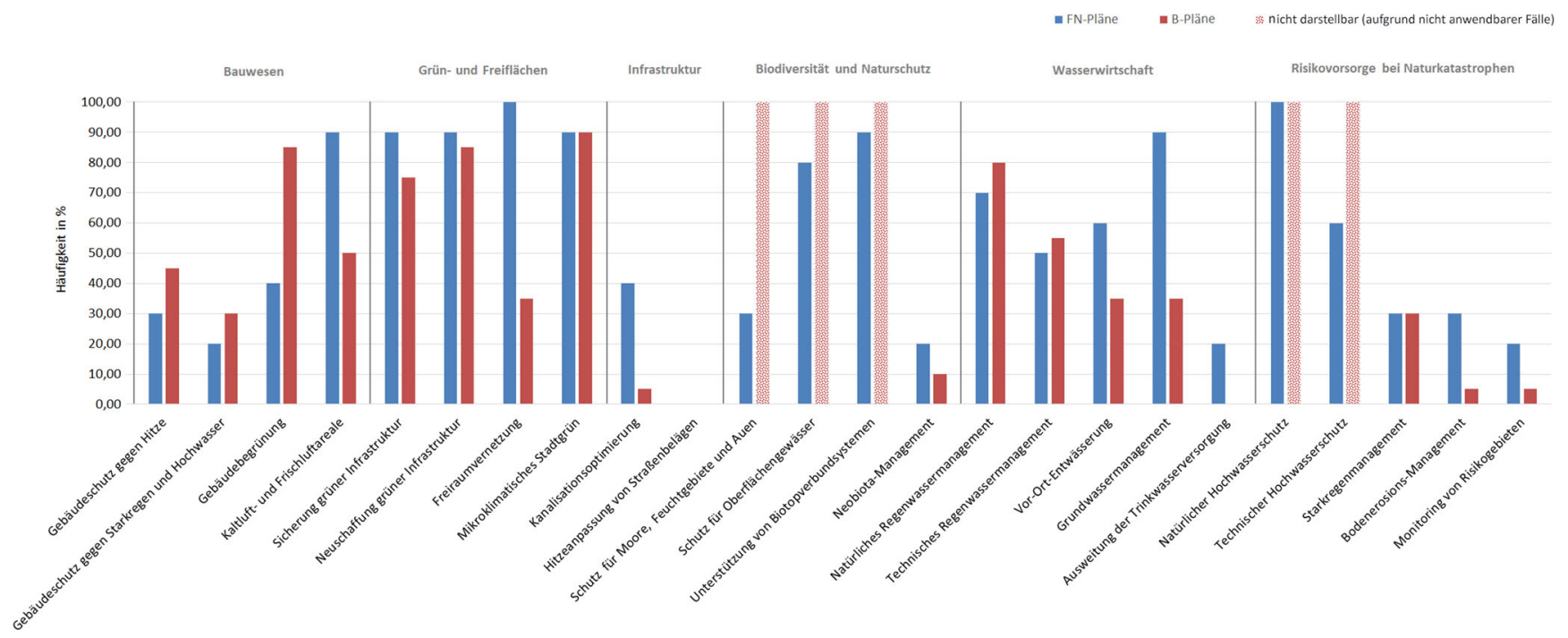

Abbildung 2 Häufigkeit der 24 untersuchten Anpassungsmaßnahmen

lungsfelder Grün- und Freiflächen, Infrastruktur, Biodiversität und Naturschutz, Wasserwirtschaft und Risikovorsorge bei Naturkatastrophen. Lediglich das Handlungsfeld Bauwesen wird stärker auf der Ebene der Bebauungspläne berücksichtigt. Außerdem ist auf beiden Planungsebenen ein deutlicher Spitzenwert bei Maßnahmen im Bereich Grünund Freiflächen zu erkennen. Auf beiden Ebenen bestehen jedoch über alle Handlungsfelder hinweg weitere Verbesserungspotenziale für die Integration von Klimaanpassung.

Eine detailliertere Auflösung jenseits der aggregierten Handlungsfelder erlaubt Abbildung 2. Sie zeigt, wie häufig die einzelnen Klimaanpassungsmaßnahmen jeweils in die beiden Bauleitplanungsebenen aufgenommen wurden. Die fünf nicht prüfbaren Maßnahmen auf der Ebene der Bebauungspläne sind hier rot schraffiert. Es bestätigt sich auch in diesem Analyseschritt der Trend, dass die Mehrheit der untersuchten Klimaanpassungsmaßnahmen deutlich häufiger in Flächennutzungsplänen Beachtung finden wird als in Bebauungsplänen (bei 11 von 19 vergleichbaren Maßnahmen). Hierzu gehören vor allem Maßnahmen mit einer größeren räumlichen Ausdehnung, wie zum Beispiel Kaltluft- und Frischluftareale, Sicherung und Neuschaffung grüner Infrastruktur und Freiraumvernetzung. Bei einem Großteil dieser Maßnahmen fallen außerdem besonders große Diskrepanzen zur Umsetzungshäufigkeit in Bebauungsplänen auf. Ein geringerer Teil der Maßnahmen zeigt die gegenläufige Verteilung, also eine verstärkte Aufnahme in die Bebauungspläne (bei 5 von 19 vergleichbaren Maßnahmen). Hierzu zählen vor allem objektspezifische oder kleinräumige Maßnahmen wie zum Beispiel Gebäudeschutz gegen Hitze sowie Starkregen und Hochwasser, Gebäudebegrünung sowie natürliches und technisches Regenwassermanagement. Bei zwei untersuchten Maßnahmen liegen beide Planungsebenen gleichauf: Mikroklimatisches Stadtgrün wurde in durch- 


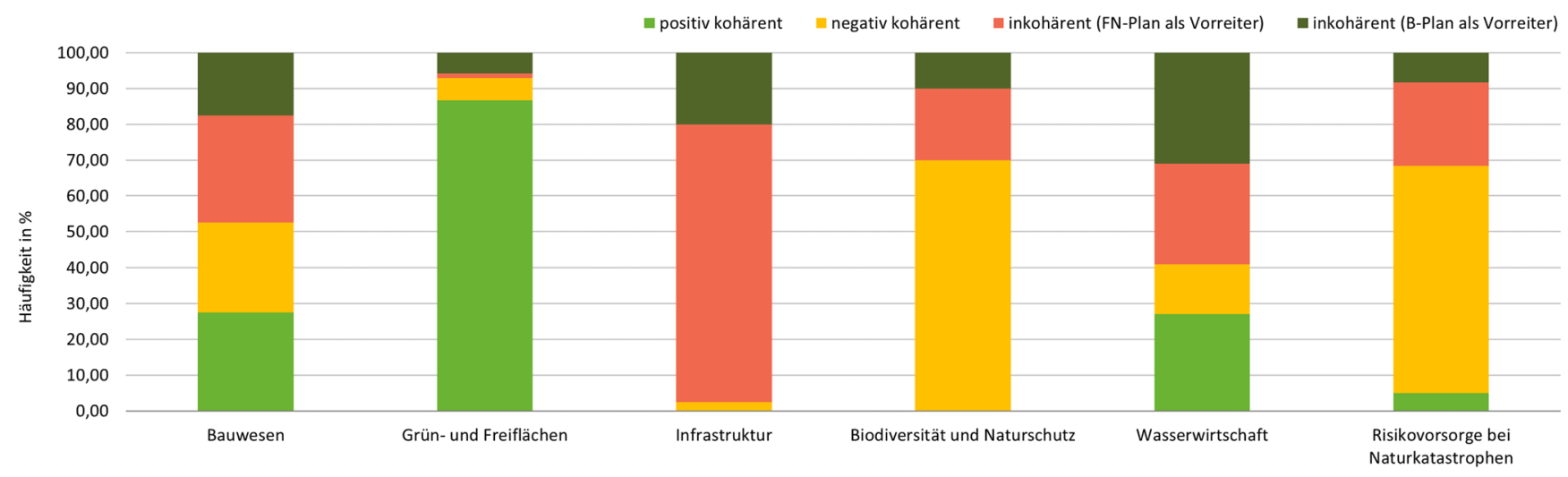

Abbildung 3 Kohärenz zwischen beiden Bauleitplanungsebenen pro Handlungsfeld

schnittlich $90 \%$ aller Pläne erwähnt. Hitzeanpassung von Straßenbelägen wurde in keinem der untersuchten Bauleitpläne genannt, wobei darauf hingewiesen sein soll, dass diese konkrete Maßnahme in der Gesamtheit aller untersuchten Flächennutzungsplänen jedoch nur in 17 von 20 Bebauungsplänen prüfbar war, während die restlichen drei Bebauungspläne keine Straßen- und Verkehrsflächen im entsprechenden Planungsgebiet beinhalten. Letzteres erklärt auch die allgemein niedrige Berücksichtigung des Handlungsfeldes Infrastruktur (vgl. Abbildung 1).

Aus diesem Analyseschritt lassen sich für beide Bauleitplanungsebenen vorranging sowie nachrangig integrierte Anpassungsmaßnahmen identifizieren. Als ,vorranging integriert" werden hier Maßnahmen definiert, welche in mindestens $80 \%$ der anwendbaren Pläne berücksichtigt werden. Als ,nachranging integriert“ werden Klimaanpassungsmaßnahmen mit einer Nennung in höchstens $20 \%$ der anwendbaren Pläne definiert. Auf Ebene der Flächennutzungspläne zählen neun von 24 zu den vorrangigen Maßnahmen, darunter zum Beispiel Kaltluft- und Frischluftareale (90\%), alle vier Maßnahmen im Handlungsfeld Grün- und Freifächen (90-100\%), natürlicher Hochwasserschutz (100\%) und Unterstützung von Biotopverbundsystemen (90\%). Zu den nachrangigen Maßnahmen in den Flächennutzungsplänen hingegen gehören fünf von 24, beispielsweise Hitzeanpassung von Straßenbelägen (0\%), Gebäudeschutz gegen Starkregen und Hochwasser (20\%), Ausweitung der Trinkwasserversorgung $(20 \%)$.

Die vorrangigen Maßnahmen auf der BebauungspläneEbene umfassen lediglich vier von 19, nämlich mikroklimatisches Stadtgrün (90\%), Gebäudebegrünung (85\%), Neuschaffung grüner Infrastruktur (85\%) sowie natürliches Regenwassermanagement $(80 \%)$. Hingegen werden auf dieser Ebene nachrangig sechs von 24 Maßnahmen berücksichtigt, hierunter ebenfalls Hitzeanpassung von Straßenbelägen $(0 \%)$, Ausweitung der Trinkwasserversorgung ( $0 \%$ ) und $\mathrm{Ka}$ nalisationsoptimierung (5\%).

\subsection{Untersuchung der Kohärenz von klimaanpassungsrelevanten Maßnahmen zwischen Flächennutzungs- und Bebauungsplänen}

Die weiterführende Frage nach der inhaltlichen Kohärenz zwischen Flächennutzungsplänen und Bebauungsplänen innerhalb der gleichen Stadt (vgl. Forschungsfrage 2) wird nachfolgend beantwortet. Diese Analyse wird allerdings begrenzt durch die Anzahl an untersuchten Bebauungsplänen, entsprechend fließen insgesamt 20 prüfbare Fälle in die Kohärenz-Untersuchung ein. Für jeden dieser 20 Fälle wurde die Kohärenz einer jeden Maßnahme $(n=19)$ ermittelt - das heißt, es wurde ermittelt, ob die Maßnahme zwischen dem untersuchten Flächennutzungsplan und Bebauungsplan positiv kohärent, negativ kohärent, inkohärent (Flächennutzungsplan als Vorreiter) oder inkohärent (Bebauungsplan als Vorreiter) ausfällt -, und anschließend über alle 20 Fälle hinweg gemittelt. Im Ergebnis zeigt sich eine mittlere Kohärenz, gemittelt über alle Maßnahmen und Städte hinweg. Es fällt auf, dass ein Großteil aller Maßnahmen, durchschnittlich $34 \%$, negativ kohärent ist, also auf keiner der beiden Planungsebenen beachtet wird. Hingegen sind $26 \%$ aller Maßnahmen positiv kohärent. Sie werden auf beiden Planungsebenen gleichsam berücksichtigt. Durchschnittlich 23\% der Maßnahmen sind inkohärent und kommen ausschließlich in den Flächennutzungsplänen (als Vorreiter) vor, jedoch nicht in den untergeordneten Bebauungsplänen. Als Gegenstück hierzu sind vergleichsweise wenige Anpassungsmaßnahmen, nämlich durchschnittlich $12 \%$, inkohärent mit einer ausschließlichen Integration in Bebauungsplänen (als Vorreiter), während sie in den übergeordneten Flächennutzungsplänen nicht vorkommen.

Abbildung 3 ermöglicht wiederum einen detaillierten Einblick in diese allgemeine Kohärenz-Verteilung und illustriert diese je Handlungsfeld. Dabei wurde für jedes Handlungsfeld und jeden Kohärenz-Fall ein arithmetischer Mit- 


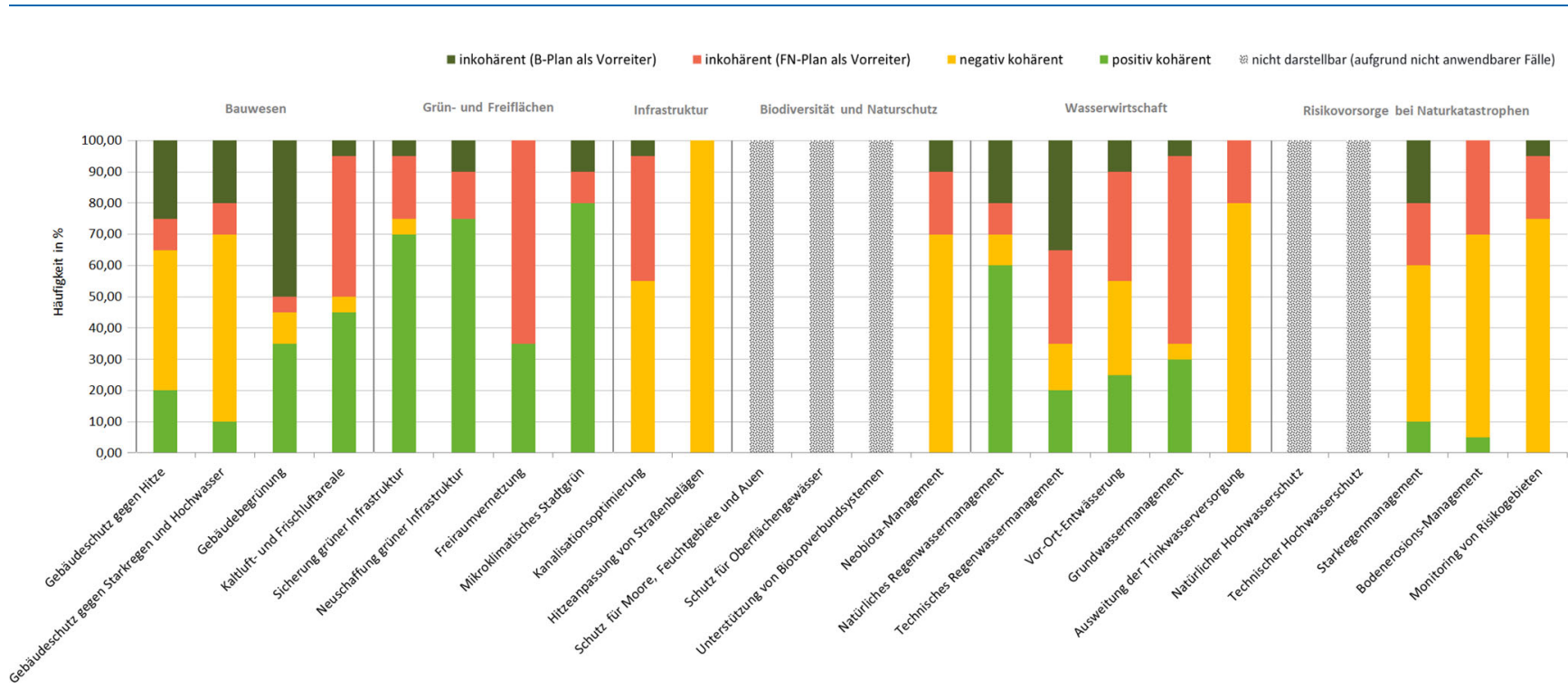

Abbildung 4 Kohärenz zwischen beiden Bauleitplanungsebenen (24 untersuchte Anpassungsmaßnahmen)

telwert über alle dem Handlungsfeld zugehörigen Maßnahmen ermittelt. So zeigen die beiden Bauleitplanungsebenen eine besonders starke Einigkeit bei der Berücksichtigung von Maßnahmen im Handlungsfeld Grün- und Freiflächen, was sich in einer sehr deutlichen positiven Kohärenz ausdrückt $(86,7 \%$, hellgrün). Darüber hinaus sind Klimaanpassungen im Handlungsfeld Infrastruktur auf beiden Planungsebenen kaum berücksichtigt ( $78 \%$, gelb), und wenn doch, so werden sie von der Ebene des Flächennutzungsplans nicht auf die Ebene des Bebauungsplans übersetzt (20\%, rot). Ähnlich unberücksichtigt bleiben Maßnahmen der Risikovorsorge bei Naturkatastrophen auf beiden Ebenen $(63 \%$, gelb). Die stärkste Inkohärenz zeigt sich im Handlungsfeld Wasserwirtschaft, wo der Flächennutzungsplan als Vorreiter für einen hohen Anteil der entsprechenden Anpassungsmaßnahmen fungiert, diese jedoch nicht in Bebauungsplänen wiederzufinden sind $(31 \%$, rot). Im Gegensatz dazu ist auffällig, dass Bebauungspläne in allen untersuchten Handlungsfeldern teilweise auch als Vorreiter auftreten, wobei besonders das Handlungsfeld Bauwesen hervorzuheben ist ( $25 \%$, dunkelgrün), wie auch schon in den vorherigen Ergebnissen gesehen. Dies bestätigt weiterhin die bereits identifizierte aktive Rolle von Bebauungsplänen im Bereich Bauwesen.

Abbildung 4 veranschaulicht einen weiteren Detailgrad für alle 19 prüfbaren Klimaanpassungsmaßnahmen: die Kohärenz-Verhältnisse zwischen den Ebenen der Flächennutzungs- und Bebauungsplanung (während die übrigen, nicht untersuchbaren fünf Maßnahmen grau schraffiert sind). Hier wurden pro Anpassungsmaßnahme alle 20 geprüften Fälle gemittelt. Im Einzelnen bestätigt sich, dass die ,beliebten “ Maßnahmen des Stadtgrüns auch die höchsten Quoten an positiver Kohärenz erreichen und entspre- chend sehr häufig inhaltlich abgestimmt auf den verschiedenen Planungsebenen der gleichen Stadt berücksichtigt werden: Sicherung grüner Infrastruktur (70\%), Neuschaffung grüner Infrastruktur (75\%) sowie mikroklimatisches Stadtgrün (80\%). Außerdem integriert der Großteil aller Fälle kohärent auf beiden Planungsebenen Maßnahmen des natürlichen Regenwassermanagements (60\%). Freiraumvernetzung hingegen wird häufig nicht auf die Ebene des Bebauungsplans überführt und verbleibt einzig in den Flächennutzungsplänen $(65 \%)$. Auf beiden Planungsebenen weitgehend bis komplett unberücksichtigt bleiben die Maßnahmen Hitzeanpassung von Straßenbelägen (100\%), Ausweitung der Trinkwasserversorgung (80\%) sowie Neobiota-Management $(70 \%)$. Hier scheinen also große Lücken innerhalb beider Bauleitplanungsebenen zu bestehen. Flächennutzungspläne fungieren meist als Vorreiter bei den Maßnahmen Freiraumvernetzung (65\%) und Grundwassermanagement $(60 \%)$. Hingegen kommt Bebauungsplänen besonders bei Maßnahmen des Gebäudeschutzes (gegen Hitze bzw. Wasser und Regen) (20 bzw. $25 \%$ ), Gebäudebegrünung (50\%) sowie natürlichem und technischem Regenwassermanagement (20 bzw. 35\%) eine Vorreiterrolle zu.

\section{Diskussion}

Aus den Ergebnissen der vorliegenden Untersuchung ist deutlich zu erkennen, dass sowohl die Ebene der Flächennutzungspläne als auch die der Bebauungspläne derzeit noch viel Potenzial zur Integration klimaanpassungsrelevanter Maßnahmen bieten. Flächennutzungspläne enthalten immerhin durchschnittlich 56\% der analysierten Anpassungs- 
maßnahmen, während es bei Bebauungsplänen nur $40 \%$ sind. Letzteres Ergebnis liegt unterhalb der modellierten Bebauungsplan-Erfolgswahrscheinlichkeit von $52 \%$ (Sprondel/Donner/Köppel 2016: 324]. Dementsprechend nutzt die Bauleitplanung aktuell noch nicht ihre konkret existierenden Handlungsspielräume aus. Diese Erkenntnisse unterstreichen die bereits bestehenden Forschungsergebnisse zur ebenfalls deutlich defizitären Integration von Klimaanpassungsbelangen in Zeitreihenanalysen von Bebauungsplänen ausgewählter Großstädte (Diepes/Müller 2018) sowie in den übergeordneten Regionalplänen (Schmitt 2016). Auch die von Buth, Kahlenborn, Savelsberg et al. (2015: 126) ermittelte Ausschöpfung der Anpassungskapazität von 52\% auf Regionalplanebene bleibt hier interessanterweise auf dem gleichen Niveau wie die Bauleitplanung. Da sowohl Regional- als auch Bauleitpläne die für sie relevanten Inhalte aus jeweils übergeordneten Plänen übernehmen müssen, könnte deren Defizit in der Integration von Anpassungsmaßnahmen auch eine geringe Priorisierung selbiger in übergeordneten Planungen erwarten lassen. Dies wäre ein interessanter Gegenstand weiterführender Untersuchungen. Wenn eine deutliche Übereinstimmung zwischen all diesen Planungsebenen nachgewiesen würde, ließe sich empfehlen, sich auf die Integration von Klimaanpassung auf der Ebene der höchsten, also der Landesplanungsebene, zu konzentrieren. Für die geeigneten Maßnahmenbereiche, also diejenigen mit der stärksten zu erwartenden Übersetzungsquote, ließe sich wiederum die in der vorliegenden Untersuchung durchgeführte Kohärenzanalyse heranziehen.

Die Analyse zur Erwähnung klimaanpassungsrelevanter Begriffe lässt vermuten, dass das Thema Klimaanpassung in Bauleitplänen nach aktuellem Stand eine untergeordnete Rolle in der planerischen Abwägung einnimmt. Während das Phänomen Hitze in 70 \% aller Bauleitpläne erwähnt wurde, erscheinen die restlichen Begriffe Klimawandel, Klimaanpassung und Starkregen überraschend isoliert und wenig erwähnt. Das deutet darauf hin, dass die Herausforderung überhitzter städtischer Bereiche ein hinlänglich bekanntes Problem darstellt und durch bestehende Instrumente bereits vielfältig Einzug in die Bauleitplanung gehalten hat, zum Beispiel angeregt durch das umweltplanerische Instrument des Landschaftsplans oder auch der Umweltprüfung und des darin behandelten Schutzgutes Klima. Die recht selten erwähnten Phänomene Klimawandel und spezifisch Starkregen sind innerhalb der Stadtplanungspraxis vergleichsweise neue Herausforderungen. Außerdem handelt es sich im Fall von Starkregen um ein flächendeckendes Klimarisiko, dessen Folgeschäden meist lokal begrenzt auftreten, was ein Grund für die geringere Auseinandersetzung innerhalb der Planungsdokumente sein könnte. Die generell niedrige Häufigkeit der begrifflichen Erwähnungen kann als Hinweis darauf interpretiert werden, dass viele der berücksichtigten
Klimaanpassungsmaßnahmen in den Planungsdokumenten nicht zwangsläufig durch den Belang der Klimaanpassung begründet werden. Stattdessen können diese, aufgrund ihres multifunktionalen Charakters, auch durch andere Belange motiviert sein (z. B. Naturschutz). Vor dem Hintergrund, dass sich die untersuchten Fallstudienstädte durch die Veröffentlichung kommunaler Klimaanpassungskonzepte bereits als thematisch sensibilisierte Akteure positioniert haben, sind die bestehenden Lücken in der Bauleitplanung letztendlich überraschend groß. Denn gerade die hier untersuchten Bebauungspläne, welche mit großer Mehrheit erst nach der Veröffentlichung der kommunalen Klimaanpassungskonzepte erschienen sind, zeigen entgegen den Erwartungen eine noch weitaus lückenhaftere Integration entsprechender Anpassungsmaßnahmen verglichen mit den untersuchten Flächennutzungsplänen, welche allesamt bereits vor den Klimaanpassungskonzepten wirksam und folglich nicht oder kaum davon beeinflusst wurden. Entsprechend steht nicht nur die Aktivität der formellen Bauleitplanung, sondern auch die Wirksamkeit der informellen planerischen Instrumente zur Debatte. Hier stellt sich die Frage, ob es einer besseren Integration von informellen Aussagen der Klimaanpassungskonzepte in die formelle Bauleitplanung bedarf und wie diese erreicht werden kann.

Gemäß ihrer charakteristischen Maßstabsebene sind in den untersuchten Flächennutzungsplänen besonders solche Anpassungsmaßnahmen bereits stark integriert, welche eine größere räumliche Ausdehnung aufweisen (z. B. Freiraumvernetzung). Bei solchen Maßnahmen besteht folglich ein vergleichsweise geringes noch bestehendes Steigerungspotenzial. Im Gegensatz dazu zeigen die Bebauungspläne einen starken Fokus auf die Berücksichtigung von kleinräumigeren Maßnahmen, wie z. B. dem Gebäudeschutz oder einigen wasserwirtschaftlichen Maßnahmen wie dem $R e$ genwassermanagement. Herausragend stark in beiden Bauleitplanungsebenen vertreten und daher positiv zu bewerten sind sämtliche Anpassungsmaßnahmen mit direktem Bezug zu Stadtgrün. Neben den Maßnahmen im Handlungsfeld Grün- und Freiflächen sind auch Maßnahmen rund um Kaltluft- und Frischluftareale, Gebäudebegrünung, natürliches Regenwassermanagement und Hochwasserschutz sowie Biotopverbundsysteme sehr stark vertreten. Bei diesen Maßnahmen handelt es sich zum einen um sogenannte Low-regretStrategien, welche meist multifunktional sind, also mehreren Zwecken gleichzeitig dienen und reversibel und relativ unkompliziert umzusetzen sind; entsprechend können als zentrale Anker für die Integration von Klimaanpassungsmaßnahmen fungieren (Diepes/Müller 2018: 307). Zum anderen werden diese aber auch schlicht durch die zahlreichen bestehenden Naturschutzauflagen gefordert (Schmitt 2016: 19). Die Priorisierung solcher Low-regret-Maßnahmen kann man auch daran ablesen, dass laut den Ergebnis- 
sen natürliche Maßnahmen des Regenwasser- und Hochwasserschutzmanagements jeweils stark bevorzugt werden vor entsprechenden technischen Maßnahmen. Darüber hinaus haben beide Bauleitplanungsebenen große Lücken im Handlungsfeld Infrastruktur und entsprechenden Handlungsbedarf, was die Regulierung von Straßenbelägen hin zu hitzeangepassten Materialien sowie die Optimierung der Kanalisationsnetze zur Vorbereitung auf Starkregenereignisse betrifft. Es sei jedoch darauf hingewiesen, dass diese Art der Klimaanpassungsmaßnahmen womöglich alternativ in fachplanerischen Vor- und Ausführungsplanungen im Zuge konkreter Projekte formuliert werden. Beide Maßnahmen sind außerdem, vor allem während Umbaumaßnahmen in Bestandsgebieten, mit recht hohem materiellem, technischem und finanziellem Aufwand verbunden.

Die Kohärenzanalyse innerhalb jeder Fallstudienstadt zeigt zum einen auf, dass der Großteil der untersuchten Klimaanpassungsmaßnahmen auf beiden Bauleitplanungsebenen kohärent unberücksichtigt bleibt $(34 \%)$, zum anderen aber auch ein Teil auf beiden Ebenen kohärent berücksichtigt wird $(26 \%)$. Hieraus wird deutlich, dass die Bauleitplanung in Bezug auf klimaanpassungsrelevante Maßnahmen mehrheitlich kohärente Entscheidungen trifft, auch wenn diese teils negativ für die Klimaanpassungsintegration ausfallen. Die inhaltlich inkohärenten Fälle hingegen fallen überwiegend zugunsten der Flächennutzungspläne als Vorreiter-Planungsebene aus (23\%), während nur ein geringer Anteil der Maßnahmen eine Planungshoheit und eigenständige Integration von Klimaanpassung auf der Ebene der Bebauungsplanung erkennen lässt $(12 \%)$. Diese Ergebnisse unterstreichen den hierarchischen Top-down-Charakter des Planungssystems. Das bedeutet, dass die Ebene der Flächennutzungsplanung ihre anleitende Rolle, im wahrsten Sinne einer „vorbereitenden“ Bauleitplanung, derzeit zwar wahrnimmt. Zugleich deuten die Ergebnisse auf eine fehlende Übersetzung von Maßnahmen der Ebene der Flächennutzungsplanung auf die der Bebauungsplanung hin. Wie bereits erwähnt, betrifft dies großräumige Maßnahmen verhältnismäßig stark. Dies ist vor allem deshalb relevant für die Planungspraxis, da erst in den Bebauungsplänen die rechtsverbindliche Festsetzung und somit Realisierung von Klimaanpassungsmaßnahmen stattfindet. Wenn Maßnahmen daher auf der eher konzeptionellen Ebene der Flächennutzungsplanung verbleiben, verringert sich die Wahrscheinlichkeit ihrer Umsetzung insgesamt. Nichtsdestotrotz wurde am Beispiel einiger kleinräumiger und objektspezifischer Maßnahmen auch deutlich, dass sich die Ebene der Bebauungsplanung im begrenzten Rahmen sehr wohl von der übergeordneten Ebene der Flächennutzungsplanung absetzen kann und nicht gänzlich auf deren Anleitung angewiesen ist. Da diese Proaktivität der Bebauungspläne jedoch weniger struktu- rell vorgegeben ist, als vielmehr von den Gegebenheiten vor Ort, dem Wissensstand und der Motivation einzelner Akteure sowie dem jeweiligen Abwägungsprozess abhängt und entsprechend situativ funktioniert, ist eine strukturelle Stärkung der Klimaanpassungsmaßnahmen in Form von parzellenscharfen Konkretisierungsvorschlägen (z. B. durch beispielhafte Festsetzungs-Textbausteine) auf der Ebene der Flächennutzungsplanung zu empfehlen.

Die vorliegenden Ergebnisse werfen außerdem weitere Forschungsfragen auf: Besteht eine direkte Pfadabhängigkeit zwischen den mangelnden klimaanpassungsrelevanten Maßnahmen in den übergeordneten Regionalplänen und dem identifizierten Defizit innerhalb der Bauleitplanung? Welchen Stellenwert haben Klimaanpassungsmaßnahmen auf der vorgelagerten Raumordnungsebene und lassen sich von hier kausale Schlüsse auf die geringe Umsetzungsquote auf den hierarchisch folgenden Ebenen ziehen? Haben informelle Klimaanpassungskonzepte nicht die raumplanerische Übersetzbarkeit und Wirksamkeit, die notwendig ist? Wie können informelle Konzepte wirksam in den Abwägungsprozess integriert werden, sodass Belange der Klimaanpassung nachhaltig in die formelle Bauleitplanung Einzug finden? Wie kann die Übersetzung großskaliger Ziele und Anpassungsmaßnahmen von Flächennutzungsplänen auf Bebauungspläne gelingen, um die tatsächliche Maßnahmenumsetzung letztendlich auch zu gewährleisten? Und andersherum, wie können kleinräumige und objektspezifische Ziele und Anpassungsmaßnahmen, welche in Bebauungsplänen bereits identifizierbar sind, auch in den Flächennutzungsplänen vermehrt konzeptionell eingearbeitet werden, um eine stadtweite und flächendeckende Anwendung sicherzustellen? Wie verhält sich die Integration von Klimaanpassungsmaßnahmen in Bebauungspläänen in Bestandsgebieten im Gegenzug zu Neubaugebieten?

Im Rahmen dieser Untersuchung und aufgrund der geringen Anzahl an Fallstudienstädten wurde keine Gegenüberstellung verschiedener Bebauungsplanarten durchgeführt. Daher wären Untersuchungen zu folgenden Fragen interessant: Gibt es Unterschiede zwischen nutzungs- und vorhabenbezogenen Bebauungsplänen (und deren Möglichkeit zur Integration von Klimaanpassung in städtebauliche Verträge)? Gibt es Unterschiede zwischen dem Innen- und Außenbereich? Gibt es Unterschiede zwischen Plänen basierend auf verschiedenen Nutzungsarten (Gewerbegebiet, Wohngebiet etc.)? Weiterhin ist auch davon auszugehen, dass der in Kapitel 2 erläuterte limitierte Handlungsspielraum für die Umsetzung von Klimaanpassungsmaßnahmen in Mittelstädten, in gleicher, wenn nicht sogar noch stärkerer Weise, auch auf Kleinstädte zutrifft. Deren aktuellen Integrationsstand in Bezug auf Klimaanpassung zu analysieren und mit demjenigen von Mittel- und Großstädten ins 
Verhältnis zu setzen, stellt einen ebenso relevanten weiteren Forschungsbedarf dar.

Wie dies für alle Studien der Fall ist, weist auch die vorliegende Untersuchung Grenzen auf. Im Wesentlichen rühren diese aus einer relativ begrenzten Datengrundlage bezüglich der Anzahl der Fallstudienstädte und der Anzahl der untersuchten Bauleitpläne. Hieraus ergibt sich eine lediglich beispielhafte Analyse des aktuellen Standes bauleitplanerischer Integration von Klimaanpassungsmaßnahmen, die keine allgemeingültigen Aussagen zulässt. Auch das entwickelte Kategoriensystem und dessen nicht vollkommen trennscharfe Abgrenzungen können zu gewissen Verzerrungen führen. Durch die entstehenden Doppelkodierungen fallen die betroffenen Maßnahmen bei der Auswertung stärker ins Gewicht (beispielsweise Dach-, Fassaden- oder Wandbegrünung). Zugleich erfassen die vorliegenden Ergebnisse nicht, wie viele Maßnahmen (im Sinne der ,Suchworte') unter dem Sammelbegriff einer Maßnahme jeweils positiv kodiert wurden. Weiterhin müssen die gebildeten Handlungsfelder stets vor dem Hintergrund ihrer teils substanziell unterschiedlichen Maßnahmenzahl betrachtet werden. Zudem beschränkt sich die vorliegende Untersuchung auf Städte, welche sich durch informelle Klimaanpassungskonzepte bereits als aktiver Akteur positioniert haben, wodurch sich ein blinder Fleck gegenüber Städten und Gemeinden ohne derlei Konzepte ergibt. Der ausschließliche inhaltliche Fokus auf die formale Bauleitplanung lässt außerdem keine konkreten Aussagen über den Inhalt informeller Instrumente (wie kommunale Klimaanpassungskonzepte) zu, obwohl diesen eine Schlüsselrolle in der klimaanpassungsrelevanten planerischen Praxis zukommen könnte.

Nichtsdestotrotz konnte die vorliegende Untersuchung wertvolle Einblicke in den aktuellen Stand der Integration klimaanpassungsrelevanter Maßnahmen in die Bauleitplanung gewähren und den aktuellen Forschungsstand vor allem um die Dimension mittelgroßer Städte sowie um die Ebene der Flächennutzungsplanung erweitern. Anhand der Ergebnisse können bestehende planerische Erfolge, aber auch nach wie vor unausgeschöpfte Handlungsspielräume identifiziert werden, um die klimaanpassungsbezogene Planungspraxis weiterhin zu verbessern.

Förderhinweis Zuwendungsgeber: Bundesministerium für Bildung und Forschung (BMBF), Zuwendungsnummer: 01LR1709C1, Förderprogramm: Zukunftsstadt - Klimaresilienz durch Handeln in Stadt und Region

Zuwendungsgeber: Bundesministerium für Bildung und Forschung (BMBF) im Förderprogramm „Leitinitiative Zukunftsstadt“; Projekt: „ExTrass - Urbane Resilienz gegenüber extremen Wetterereignissen“ (Zuwendungsnummer: 01LR1709C1)

\section{Literatur}

Albrecht, J.; Schanze, J.; Klimmer, L.; Bartel, S. (2018): Klimaanpassung im Raumordnungs-, Städtebau- und Umweltfachplanungsrecht sowie im Recht der kommunalen Daseinsvorsorge. Grundlagen, aktuelle Entwicklungen und Perspektiven. Dessau-Roßlau. = Climate Change 03.

Baumüller, N. (2018): Stadt im Klimawandel. Klimaanpassung in der Stadtplanung. Grundlagen, Maßnahmen und Instrumente. Dissertation am Städtebau-Institut der Universität Stuttgart. https://doi.org/10.18419/opus9821

BMVBS - Bundesministerium für Verkehr, Bau und Stadtentwicklung (Hrsg.) (2013a): Flexibilisierung der Planung für eine klimawandelgerechte Stadtentwicklung. Berlin. = BMVBS-Online-Publikation 16.

BMVBS - Bundesministerium für Verkehr, Bau und Stadtentwicklung (Hrsg.) (2013b): Planungsbezogene Empfehlungen zur Klimaanpassung auf Basis der Maßnahmen des Stadtklimalotsen. Berlin. = BMVBS-Online-Publikation 25 .

BMVBS - Bundesministerium für Verkehr, Bau und Stadtentwicklung (Hrsg.) (2013c): Methodenhandbuch zur regionalen Klimafolgenbewertung in der räumlichen Planung. Systematisierung der Grundlagen regionalplanerischer Klimafolgenbewertung. Berlin.

Bubeck, P.; Klimmer, L.; Albrecht, J. (2016): Klimaanpassung in der rechtlichen Rahmensetzung des Bundes und Auswirkungen auf die Praxis im Raumordnungs-, Städtebau- und Wasserrecht. In: Natur und Recht 38, 5, 297-307. https://doi.org/10.1007/s10357-016-3003-1

Bundesregierung (2008): Deutsche Anpassungsstrategie an den Klimawandel, vom Bundeskabinett am 17. Dezember 2008 beschlossen. Berlin.

Bundesregierung (2011): Aktionsplan Anpassung der Deutschen Anpassungsstrategie an den Klimawandel, vom Bundeskabinett am 31. August 2011 beschlossen. Berlin.

Bundesregierung (2015): Fortschrittsbericht zur Deutschen Anpassungsstrategie an den Klimawandel. Berlin.

Buth, M.; Kahlenborn, W.; Savelsberg, J.; Becker, N.; Bubeck, P.; Kabisch, S.; Kind, C.; Tempel, A.; Tucci, F.; Greiving, S.; Fleischhauer, M.; Lindner, C.; Lückenkötter, J.; Schonlau, M.; Schmitt, H.; Hurth, F.; Othmer, F.; Augustin, R.; Becker, D.; Abel, M.; Bornemann, T.; Steiner, H.; Zebisch, M.; Schneiderbauer, S.; Kofler, C. (2015): Vulnerabilität Deutschlands gegenüber dem Klimawandel. Dessau-Roßlau. = Climate Change 24.

Diepes, C. J.; Müller, N. D. (2018): Klimarelevante Handlungsfelder in der verbindlichen Bauleitplanung - Nutzen deutsche Großstädte den ihnen zur Verfügung ste- 
henden Handlungsspielraum für Klimaschutz und Klimaanpassung aus? In: Zeitschrift für Umweltpolitik und Umweltrecht 31, 3, 288-323.

Fleischhauer, M.; Bornefeld, B. (2006): Klimawandel und Raumplanung. Ansatzpunkte der Raumordnung und Bauleitplanung für den Klimaschutz und die Anpassung an den Klimawandel. In: Raumforschung und Raumordnung 64, 3, 161-171. https://doi.org/10.1007/ BF03182977

Frommer, B.; Schlipf, S.; Böhm, H. R.; Janssen, G.; Sommerfeldt, P. (2013): Die Rolle der räumlichen Planung bei der Anpassung an die Folgen des Klimawandels. In: Birkmann, J.; Vollmer, M.; Schanze, J. (Hrsg.): Raumentwicklung im Klimawandel. Herausforderungen für die räumliche Planung. Hannover, 120-148. = Forschungsberichte der ARL 2.

Hasse, J.; Willen, L.; Baum, N.; Bongers-Römer, S.K.; Pichl, J.; Völker, V. (2019): Umfrage Wirkung der Deutschen Anpassungsstrategie (DAS) für die Kommunen: Teilbericht. Dessau-Roßlau. = Climate Change 01 .

Mayring, P.; Fenzl, T. (2014): Qualitative Inhaltsanalyse. In: Baur, N.; Blasius, J. (Hrsg.): Handbuch Methoden der empirischen Sozialforschung. Wiesbaden. 543-556. https://doi.org/10.1007/978-3-531-18939-0_38

MKRO - Ministerkonferenz für Raumordnung (2009): Bericht des Hauptausschusses der Ministerkonferenz für Raumordnung (MKRO). Handlungskonzept der Raumordnung zu Vermeidungs-, Minderungs- und Anpassungsstrategien im Hinblick auf die räumlichen Konsequenzen des Klimawandels. Berlin.

Müller-Ibold, K. (1996): Einführung in die Stadtplanung. Band 2: Leitgedanken, Systeme und Strukturen. Stuttgart.

Rabe, K.; Pauli, F.; Wenzel, G. (2014): Bau- und Planungsrecht: Raumordnungs- und Landesplanungsrecht, Allgemeines Städtebaurecht, Städtebauliche Sanierung und Entwicklung, Bauordnungsrecht, Bauaufsichtliche Maßnahmen, Baurechtlicher Nachbarschutz. Stuttgart.
Reckien, D.; Flacke, J.; Dawson, R. J.; Heidrich, O.; Olazabal, M.; Foley, A.; Hamann, J. J.-P.; Orru, H.; Salvia, M.; de Gregorio Hurtado, S.; Geneletti, D.; Pietrapertosa, F. (2014): Climate change response in Europe: what's the reality? Analysis of adaptation and mitigation plans from 200 urban areas in 11 countries. In: Climatic Change 122, 1-2, 331-340. https://doi.org/10.1007/ s10584-013-0989-8

Schmitt, H. C. (2014): Analyse der Anpassungskapazität der Regionalplanung an den Klimawandel. Masterarbeit an der Technischen Universität Dortmund.

Schmitt, H. C. (2016): Klimaanpassung in der Regionalplanung - Eine deutschlandweite Analyse zum Implementationsstand klimaanpassungsrelevanter Regionalplaninhalte. In: Raumforschung und Raumordnung 74, 1, 9-21. https://doi.org/10.1007/s13147-015-0375-2

Sprondel, N. F.; Donner, J.; Köppel, J. (2016): Klimaanpassung im Bebauungsplan: Eignung von Bayesian Networks zur Ermittlung der Wahrscheinlichkeit einer Implementierung von Maßnahmen. In: Naturschutz und Landschaftsplanung 48, 10, 321-327.

Streich, B. (2011): Stadtplanung in der Wissensgesellschaft: Ein Handbuch. Wiesbaden. https://doi.org/10.1007/9783-531-93164-7

UBA - Umweltbundesamt (Hrsg.) (2016): Praxishilfe Klimaanpassung in der räumlichen Planung. Raum- und fachplanerische Handlungsoptionen zur Anpassung der Siedlungs- und Infrastrukturen an den Klimawandel. Dessau-Roßlau.

Weidlich, S. (2020): Anpassung an die Folgen des Klimawandels. Rechtliche Steuerung bei unsicheren Wissensgrundlagen. Kassel. = Interdisciplinary Research on Climate Change Mitigation and Adaptation 14.

Zimmermann, K.; Boghrat, J.; Weber, M. (2015): The epistemologies of local climate change policies in Germany. In: Urban Research and Practice 8, 3, 303-318. https:// doi.org/10.1080/17535069.2015.1051379 